\title{
Parity Breaking Medium and Squeeze Operators
}

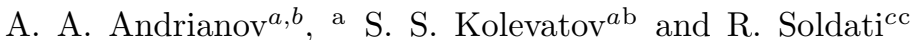 \\ a Saint-Petersburg State University, 7/9 Universitetskaya nab., St.Petersburg, Russia \\ ${ }^{b}$ Institut de Ciències del Cosmos (ICCUB), Universitat de Barcelona, Barcelona, Catalonia, Spain and \\ c Dipartimento di Fisica e Astronomia - Universitá di Bologna \\ and Istituto Nazionale di Fisica Nucleare - Sezione di Bologna, Italy
}

The transition between a Minkowski space region and a parity breaking medium domain is thoroughly discussed. The requirement of continuity of the field operator content across the separating boundary of the two domains leads to Bogolyubov transformations, squeezed pairs states and squeeze operators that turn out to generate a functional SU(2) algebra. According to this algebraic approach, the reflection and transmission probability amplitude across the separating boundary are computed. The probability rate of the emission or absorption of squeezed pairs out of the vacuum (generalization of the Sauter-Schwinger-Nikishov formula) is obtained.

PACS numbers: 11.10.-z,11.15.Yc,12.20.-m

\footnotetext{
${ }^{a}$ Corresponding author: a.andrianov@spbu.ru

b s.s.kolevatov@gmail.com

c roberto.soldati@bo.infn.it
} 


\section{INTRODUCTION: POSSIBLE PHYSICS OF TIME- OR SPACE-DEPENDENT PSEUDOSCALAR CONDENSATE}

During the last decades the available bounds for validity of fundamental laws in Physics have been attracting more and more attention following succeeding experimental improvements, both in the laboratory research and in astrophysics [1]-6]. More specifically, in Quantum Electrodynamics the interest towards possible Lorentz and CPT Invariance Violations (LIV for short) was raised up after the seminal paper [1], where the very possibility to deal with a parity odd vector background in the large scale Universe was conjectured. The latter was employed to modify QED by supplementing it with the Chern-Simons (CS for short) term in the Action. Later on the various aspects of its signatures were discussed [7]-[17] although it has not yet been detected [18]-[20].

In particular, spontaneous Lorentz symmetry breaking may cause LIV after condensation of massless axion-like fields [7], [18]- 23]. Cold relic axions resulting from vacuum misalignment [24, 25] in the early Universe is a popular and so far viable candidate to dark matter. If we assume that cold axions are the only contributors to the matter density of the Universe apart from ordinary baryonic matter its density must be [26] of the order

$$
\rho \simeq 10^{-30} \mathrm{~g} \mathrm{~cm}^{-3} \simeq 10^{-46} \mathrm{GeV}^{4}
$$

Of course dark matter is not uniformly distributed, its distribution traces that of visible matter (or rather the other way round). As well, on stellar scales, the emergence of spontaneous Lorentz symmetry breaking in bubbles of pseudo-scalar condensates (of pions or axions) may be detected in neutron stars making influence on their cooling rate [27].

Last decade several experiments in heavy ion collisions have indicated an abnormal yield of lepton pairs of invariant mass $<1 \mathrm{GeV}$ in the region of small rapidity and moderate transverse momenta [28 30] (reviewed in [31]). This effect is visible only for collisions that are central or nearly central. Most studies refer to $e^{+} e^{-}$pairs but dimuon pairs have also been found to be produced in excess above the dimuon threshold. The explanation of this enhancement is outlined in 32] complementing the more conventional thermal effects. We conjecture that the effect may be a manifestation of local parity breaking in colliding nuclei due to generation of pseudo-scalar, isosinglet or neutral isotriplet, classical background whose magnitude and profile depends on the dynamics of the collision. Recently a possibility to generate an isotriplet pseudo-scalar condensate at large baryon densities has been argued for in [33]. In 34] it has been suggested that for peripheral interactions a complementary effect should occur, namely, an isosinglet pseudo-scalar background could appear as the result of large-scale fluctuation of topological charge leading to the so-called Chiral Magnetic Effect (CME) studied also by lattice QCD simulations [35] and seemingly detected in the STAR experiments on RHIC [36].

All above mentioned phenomena are situated in bounded volumes and a specific parity violating effect, namely, the gradient of isosinglet pseudo-scalar condensate can be formed near the volume boundary separating from a parity-even Maxwell QED vacuum. In the static situation this gradient is orthogonal to the boundary and it may generate spatial parity breaking in QED interacting to photons or more generally to vector mesons via the Chern-Simons interaction presumably induced by fermion polarization effects [16].

In this paper we present a thorough analysis of the quantum theory of massive vector fields on a flat space-time in the presence of a boundary. Specifically, the boundary corresponds to a three-dimensional hyper-plane which separates two half-spaces of the Minkowski space, on one side of which a Chern-Simons interaction is active. We restrict ourselves with the CS dynamics generated by a CS vector orthogonal to the flat boundary which perfectly guaranties the gauge invariance while provides the Lorentz symmetry violation in the Maxwell-Chern-Simons part coherent with boundary implementation. On the other half-space we have a massive quantum vector field in vacuum. Sect. 2 is devoted to definitions: namely, real massive Abelian vector fields are put within a pseudo-scalar background with a constant gradient on an half-space, while in the complementary half-space they propagate in an empty Minkowski space. Then the quantum theory for such a kind of configuration for the vector fields is briefly outlined. In Sect. 3 the Bogolyubov transformations connecting the operator algebras of creation and destruction operators are derived (in a full analogy with their usage for quantization of matter in curved spaces or accelerated frames, see [37]), which are related to the presence of a boundary between a parity breaking Chern-Simons background medium and an empty Minkowski space. In Sect. 4 the functional squeeze operator algebra is obtained and discussed, which allows for a description and implementation of the transmission and reflection through a boundary in terms of a purely algebraic approach. A short description of this algebra was presented in [38]. In Sect. 5 the rate of squeezed pairs emission and absorption in the presence of a parity breaking Chern-Simons background medium is evaluated, i.e. the calculation of the probability to find any number of squeezed pairs of vector particles of the Proca-Stückelberg or Chern-Simons kind in crossing the boundary between the empty space-time and the parity breaking medium is performed. It is an extension of the Sauter-Schwinger-Nikishov formula 39]-[41]. The Appendix contains some further technical details concerning the above mentioned computation of the emission-absorption rate. Throughout this paper we shall use a 
Minkowski metric tensor $g_{\mu \nu}=g^{\mu \nu}=\operatorname{diag}(+1,-1,-1,-1)$ and a natural system of units $\hbar=c=1$, unless explicitly stated.

\section{MASSIVE VECTOR FIELDS IN A PSEUDO-SCALAR BACKGROUND}

We start from the Stückelberg-type Lagrangian [42, 43] which describes the propagation of an Abelian massive real vector field in the presence of a pseudo-scalar, or axion-like, background field [1], viz.,

$$
\begin{aligned}
\mathcal{L} & =-\frac{1}{4} F^{\alpha \beta}(x) F_{\alpha \beta}(x)-\frac{1}{4} g \eta_{c \ell}(x) F^{\mu \nu}(x) \widetilde{F}_{\mu \nu}(x) M^{-1} \\
& +\frac{1}{2} m^{2} A_{\nu}(x) A^{\nu}(x)+A^{\mu}(x) \partial_{\mu} B(x)+\frac{1}{2} \varkappa B^{2}(x)
\end{aligned}
$$

where $A^{\mu}$ and $\eta_{c \ell}$ stand for the vector and background pseudo-scalar fields respectively, $\widetilde{F}^{\mu \nu}=\frac{1}{2} \varepsilon^{\mu \nu \rho \sigma} F_{\rho \sigma}$ is the dual field strength, while $B$ is the auxiliary Stückelberg scalar field [42] with $\varkappa \in \mathbb{R}$. The positive dimensionless coupling $g>0$ and the (large) mass parameter $M \gg m$ do specify the intensity and the scale of the pseudo-scalar-vector interaction. For example, axions or axion-like particles arise generically from string-like models or Standard Model extensions, with a natural size of the decay constant $M$, often also denoted by $f_{a}$, typically varying between $10^{9}$ and $10^{17} \mathrm{GeV}$ [44], a pretty large scale that will be suitably used and recognized in the sequel as an effective UV cut-off regulator which characterizes the adiabatic approximation of a constant LIV background vector $\zeta^{\mu}$ as we shall see hereafter. Notice that we have included the Proca mass term for the vector field because, as it is discussed in [32], the latter is required to account for the strong interaction effects in heavy ions collisions supported by massive vector mesons $(\rho, \omega, \ldots)$ in addition to photons. Moreover, as thoroughly debated in [16, 17], the mass term for the vector field appears to be generally necessary to render the dynamics self-consistent in the presence of a Chern-Simons Lagrangian and is actually induced by the radiative corrections from the LIV fermionic matter. The auxiliary part of the Stückelberg Lagrangian, which further violates gauge invariance beyond the mass term for the vector field, has been introduced to provide - just owing to the renowned Stückelberg trick - the simultaneous occurrences of power counting renormalizability and perturbative unitarity for a general interacting theory. Its presence allows for a smooth mass-less limit of the quantum vector field. Here we shall consider the adiabatic limit of a slowly varying classical pseudo-scalar background of the kind

$$
\eta_{c \ell}(x) \simeq \frac{M}{g} \zeta_{\lambda} x^{\lambda} \theta(-\zeta \cdot x)
$$

where $\theta(\cdot)$ is the Heaviside step distribution, in which a fixed constant four vector $\zeta^{\mu}$ with the dimensions of an inverse length has been introduced, in a way to violate Lorentz and CPT invariances in the Minkowski half-space $\zeta \cdot x<0$. In what follows we shall suppose that $\zeta^{2} \neq 0$. If we now insert the specific form (3) of the pseudo-scalar background in the pseudo-scalar-vector coupling Lagrangian we can write

$$
\begin{aligned}
-\frac{1}{4} F^{\mu \nu}(x) \widetilde{F}_{\mu \nu}(x) \zeta_{\lambda} x^{\lambda} \theta(-\zeta \cdot x) & =\frac{1}{2} \zeta_{\mu} A_{\nu}(x) \widetilde{F}^{\mu \nu}(x) \theta(-\zeta \cdot x) \\
& -\partial_{\mu}\left[\frac{1}{2} A_{\nu}(x) \widetilde{F}^{\mu \nu}(x) \zeta_{\lambda} x^{\lambda} \theta(-\zeta \cdot x)\right]
\end{aligned}
$$

The very last term in the RHS of the above equality is evidently a boundary term, its contribution to the Action being reduced for the Gauß theorem to

$$
\int_{\Omega} \mathrm{d}^{4} x \partial_{\mu}\left[\frac{1}{2} A_{\nu}(x) \widetilde{F}^{\mu \nu}(x) \zeta_{\lambda} x^{\lambda} \theta(-\zeta \cdot x)\right]=\frac{1}{2} \int_{\partial \Omega} \mathrm{d} \sigma_{\mu} A_{\nu}(x) \widetilde{F}^{\mu \nu}(x) \zeta_{\lambda} x^{\lambda} \theta(-\zeta \cdot x)
$$

where $\Omega$ is an arbitrary domain of the Minkowski space that is bounded by the initial and final three dimensional space-like oriented surfaces $\partial \Omega=\Sigma_{\imath} \cup \Sigma_{f}$. Hence the boundary term won't contribute to the Euler-Lagrange field equations iff

$$
\left.A_{\nu}(x) \widetilde{F}^{\mu \nu}(x) \zeta_{\lambda} x^{\lambda} \theta(-\zeta \cdot x)\right|_{\Sigma_{\imath}}=\left.A_{\nu}(x) \widetilde{F}^{\mu \nu}(x) \zeta_{\lambda} x^{\lambda} \theta(-\zeta \cdot x)\right|_{\Sigma_{f}} \equiv 0
$$

which entails a particular fall down of the vector potential and field strength for large space-like separations in the half-space $\zeta \cdot x<0$. In such a circumstance we can derive the field equations from the equivalent Lagrangian

$$
\begin{aligned}
\mathcal{L} & =-\frac{1}{4} F^{\alpha \beta}(x) F_{\alpha \beta}(x)+\frac{1}{2} \zeta_{\mu} A_{\nu}(x) \widetilde{F}^{\mu \nu}(x) \theta(-\zeta \cdot x) \\
& +\frac{1}{2} m^{2} A_{\nu}(x) A^{\nu}(x)+A^{\mu}(x) \partial_{\mu} B(x)+\frac{1}{2} \varkappa B^{2}(x)
\end{aligned}
$$


in which the gauge invariance is explicitly broken by all the terms but the first one, i.e. the Maxwell's Lagrangian for the radiation fields. Then the Euler-Lagrange field equations read

$$
\left\{\begin{array}{cc}
\partial_{\lambda} F^{\lambda \nu}+m^{2} A^{\nu}+\zeta_{\alpha} \widetilde{F}^{\alpha \nu}+\partial^{\nu} B=0 & \text { for } \zeta \cdot x<0 \\
\partial_{\lambda} F^{\lambda \nu}+m^{2} A^{\nu}+\partial^{\nu} B=0 & \text { for } \zeta \cdot x>0 \\
\partial_{\nu} A^{\nu}=\varkappa B &
\end{array}\right.
$$

After contraction of the first pair of the above set of field equations with $\partial_{\nu}$ we find

$$
\left(\square+\varkappa m^{2}\right) B(x)=0
$$

whence it follows that the auxiliary Stückelberg field is always a decoupled non-physical real scalar field, which is never affected by the pseudo-scalar classical background $\forall \varkappa \in \mathbb{R}$. From now on we shall select the simplest choice $\varkappa=1$ that leads to the Klein-Gordon equation for the auxiliary field, together with

$$
\left\{\begin{array}{cc}
\square A^{\nu}(x)+m^{2} A^{\nu}(x)=\varepsilon^{\nu \alpha \rho \sigma} \zeta_{\alpha} \partial_{\rho} A_{\sigma}(x) & \text { for } \zeta \cdot x<0 \\
\square A^{\nu}(x)+m^{2} A^{\nu}(x)=0 & \text { for } \zeta \cdot x>0 \\
\partial_{\nu} A^{\nu}(x)=B(x) & \left(\square+m^{2}\right) B(x)=0
\end{array}\right.
$$

In order to find the general solution of the above linear equations (6) we turn to the momentum space

$$
A^{\nu}(x)=\int \frac{\mathrm{d}^{4} k}{(2 \pi)^{3 / 2}} \mathrm{a}^{\nu}(k) \mathrm{e}^{-i k \cdot x} \quad B(x)=\int \frac{\mathrm{d}^{4} k}{(2 \pi)^{3 / 2}} \mathrm{~b}(k) \mathrm{e}^{-i k \cdot x}
$$

so that

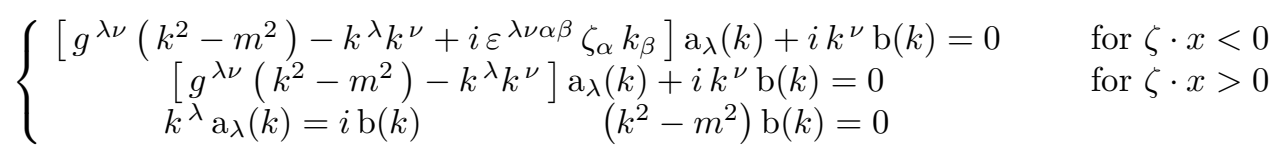

The general solution for the auxiliary field operator is well known, viz.,

$$
\begin{array}{r}
B(x)=\int \mathrm{d} \mathbf{k}\left[\mathrm{b}_{\mathbf{k}} u_{\mathbf{k}}(x)+\mathrm{b}_{\mathbf{k}}^{\dagger} u_{\mathbf{k}}^{*}(x)\right] \\
u_{\mathbf{k}}(x)=\left[(2 \pi)^{3} 2 k_{0}\right]^{-1 / 2} \exp \left\{-i k_{\nu} x^{\nu}\right\} \quad k_{0}=\sqrt{\mathbf{k}^{2}+m^{2}}
\end{array}
$$

with ghost-like canonical commutation relations

$$
\left[\mathrm{b}_{\mathbf{k}}^{\dagger}, \mathrm{b}_{\mathbf{p}}\right]=\delta(\mathbf{k}-\mathbf{p}) \quad\left[\mathrm{b}_{\mathbf{k}}, \mathrm{b}_{\mathbf{p}}\right]=0
$$

The general solutions on the two half-spaces separated by the hyper-plane $\zeta \cdot x=0$ have to be set in a more convenient form to the purpose of our discussion. It turns out that the Proca-Stückelberg and the Maxwell-Chern-Simons massive vector fields face one each other at the boundary $\zeta \cdot x=0$. Hence, continuity of the quantum fields does require equality on the surface separating the parity breaking medium from the Minkowski space: namely,

$$
\delta(\zeta \cdot x)\left[A_{\mathrm{PS}}^{\mu}(x)-A_{\mathrm{CS}}^{\mu}(x)\right]=0
$$

while the auxiliary non-physical field $B(x)$ is by no means affected by the presence of the hyper-plane $\zeta \cdot x=0$, as already noticed.

\section{A. The Proca-Stückelberg Quantum Field in the Presence of a Boundary}

On the half-space $\zeta \cdot x>0$, the general solutions of the field equations are the well known Proca-Stückelberg vector and auxiliary ghost scalar free quantum fields. In order to write the general solutions in the presence of the infinite hyper-plane separation boundary $\zeta \cdot x=0$, from now on it is convenient to use a slightly different notation: namely, we set $\mathrm{x}=\left(x_{0}, x^{1}, x^{2}, x^{3}\right)=(t, x, y, z)$ in natural units. Consider the case of the spatial Chern-Simons constant vector $\zeta^{\mu}=(0, \zeta, 0,0)$ with $\zeta>0$ and the corresponding space-like hyper-plane $x^{1} \equiv x=0$, in such a manner that $\delta(\zeta \cdot \mathrm{x})=\zeta^{-1} \delta(x)$. Let us define the following quantities:

$$
\hat{\mathrm{k}} \equiv\left(k_{0}, k_{y}, k_{z}\right) ; \quad \hat{\mathrm{x}} \equiv(t, y, z) ; \quad \hat{\mathrm{k}} \cdot \hat{\mathrm{x}}=t k_{0}-y k_{y}-z k_{z} ; \quad k_{0}=\omega_{\mathbf{k}} \equiv \sqrt{\mathbf{k}^{2}+m^{2}}
$$


Then we can write the Proca-Stückelberg solution in form

$$
\begin{aligned}
A_{\mathrm{PS}}^{\mu}(x, \hat{\mathrm{x}}) & =\int \mathrm{d} \mathbf{k} \sum_{r=1}^{3}\left[\mathrm{a}_{\mathbf{k}, r} u_{\mathbf{k}, r}^{\mu}(x, \hat{\mathrm{x}})+\mathrm{a}_{\mathbf{k}, r}^{\dagger} u_{\mathbf{k}, r}^{\mu *}(x, \hat{\mathrm{x}})\right] \\
u_{\mathbf{k}, r}^{\nu}(x, \hat{\mathrm{x}}) & =\theta(-x)\left[(2 \pi)^{3} 2 \omega_{\mathbf{k}}\right]^{-1 / 2} e_{r}^{\nu}(\mathbf{k}) \exp \{i K x-i \hat{\mathrm{k}} \cdot \hat{\mathrm{x}}\} ; \quad(r=1,2,3)
\end{aligned}
$$

with $x \leq 0, K \equiv k^{1}=k_{x}$, where the creation destruction operators fulfill the canonical commutation relations

$$
\left[\mathrm{a}_{\mathbf{k}, r}, \mathrm{a}_{\mathbf{k}^{\prime}, s}^{\dagger}\right]=\delta\left(\mathbf{k}-\mathbf{k}^{\prime}\right) \delta_{r s}
$$

all the remaining commutators being equal to zero. The three linear polarization real vectors do satisfy the orthogonality and closure relations on the mass shell $K^{2}=\hat{\mathrm{k}}^{2}-m^{2}$ : namely,

$$
\mathrm{k}_{\mu} e_{r}^{\mu}(\mathbf{k})=0 ; \quad-g_{\mu \nu} e_{r}^{\mu}(\mathbf{k}) e_{s}^{\nu}(\mathbf{k})=\delta_{r s} ; \quad \sum_{r=1}^{3} e_{r}^{\mu}(\mathbf{k}) e_{r}^{\nu}(\mathbf{k})=-g^{\mu \nu}+\frac{\mathrm{k}^{\mu} \mathrm{k}^{\nu}}{m^{2}}
$$

It is physically motivated to split the Proca-Stückelberg vector field into the so called progressive (or incident) and regressive (or reflected) parts, that corresponds to positive and negative longitudinal momenta $K=k^{1}=k_{x} \gtrless 0$ respectively: namely,

$$
\begin{aligned}
& A_{\rightarrow}^{\mu}(x, \hat{\mathrm{x}}) \equiv \int_{0}^{\infty} \mathrm{d} K \int_{-\infty}^{\infty} \mathrm{d} k_{y} \int_{-\infty}^{\infty} \mathrm{d} k_{z} \sum_{r=1}^{3}\left[\mathrm{a}_{\mathbf{k}, r} u_{\mathbf{k}, r}^{\mu}(x, \hat{\mathrm{x}})+\mathrm{a}_{\mathbf{k}, r}^{\dagger} u_{\mathbf{k}, r}^{\mu *}(x, \hat{\mathrm{x}})\right] \\
& A_{\leftarrow}^{\nu}(x, \hat{\mathrm{x}}) \equiv \int_{0}^{\infty} \mathrm{d} K \int_{-\infty}^{\infty} \mathrm{d} k_{y} \int_{-\infty}^{\infty} \mathrm{d} k_{z} \sum_{r=1}^{3}\left[\mathrm{a}_{-\mathbf{k}, r} u_{-\mathbf{k}, r}^{\nu}(x, \hat{\mathrm{x}})+\mathrm{a}_{-\mathbf{k}, r}^{\dagger} u_{-\mathbf{k}, r}^{\nu *}(x, \hat{\mathrm{x}})\right]
\end{aligned}
$$

where we understand $-\mathbf{k} \equiv\left(-K, k_{y}, k_{z}\right)$. Of course we have

$$
\begin{gathered}
u_{-\mathbf{k}, r}^{\nu}(x, \hat{\mathrm{x}})=\theta(-x)\left[(2 \pi)^{3} 2 k_{0}\right]^{-1 / 2} e_{r}^{\nu}(-\mathbf{k}) \exp \left\{-i K x+i y k_{y}+i z k_{z}-i t k_{0}\right\} \\
\equiv \bar{u}_{\mathbf{k}, r}^{\nu}(x, \hat{\mathrm{x}}) \\
{\left[\mathrm{a}_{-\mathbf{k}, r}, \mathrm{a}_{\mathbf{k}, s}^{\dagger}\right]=\left[\mathrm{a}_{-\mathbf{k}, r}, \mathrm{a}_{\mathbf{k}, s}\right]=0}
\end{gathered}
$$

in such a manner that if we set

$$
\mathrm{a}_{-\mathbf{k}, r} \equiv \overline{\mathrm{a}}_{\mathbf{k}, r} \quad \forall \mathbf{k}=\left(K, k_{y}, k_{z}\right) \quad \text { with } \quad K>0, k_{y}, k_{z} \in \mathbb{R}
$$

we can eventually write the normal modes expansions for $x \leq 0$

$$
\begin{aligned}
& A_{\rightarrow}^{\mu}(x, \hat{\mathrm{x}}) \equiv \int_{0}^{\infty} \mathrm{d} K \int_{-\infty}^{\infty} \mathrm{d} k_{y} \int_{-\infty}^{\infty} \mathrm{d} k_{z} \sum_{r=1}^{3}\left[\mathrm{a}_{\mathbf{k}, r} u_{\mathbf{k}, r}^{\mu}(x, \hat{\mathrm{x}})+\mathrm{a}_{\mathbf{k}, r}^{\dagger} u_{\mathbf{k}, r}^{\mu *}(x, \hat{\mathrm{x}})\right] \\
& A_{\leftarrow}^{\nu}(x, \hat{\mathrm{x}}) \equiv \int_{0}^{\infty} \mathrm{d} K \int_{-\infty}^{\infty} \mathrm{d} k_{y} \int_{-\infty}^{\infty} \mathrm{d} k_{z} \sum_{r=1}^{3}\left[\overline{\mathrm{a}}_{\mathbf{k}, r} \bar{u}_{\mathbf{k}, r}^{\nu}(x, \hat{\mathrm{x}})+\overline{\mathrm{a}}_{\mathbf{k}, r}^{\dagger} \bar{u}_{\mathbf{k}, r}^{\nu *}(x, \hat{\mathrm{x}})\right]
\end{aligned}
$$

where

$$
\left[\overline{\mathrm{a}}_{\mathbf{k}, r}, \mathrm{a}_{\mathbf{k}^{\prime}, s}^{\dagger}\right]=\left[\overline{\mathrm{a}}_{\mathbf{k}, r}, \mathrm{a}_{\mathbf{k}^{\prime}, s}\right]=0
$$

so that

$$
\left[A_{\rightarrow}^{\mu}(x, \hat{\mathrm{x}}), A_{\leftarrow}^{\nu}\left(x^{\prime}, \hat{\mathrm{x}}^{\prime}\right)\right]=0
$$

Now it is expedient to change the integration variable into the normal modes expansions (18) 19 from $K>0$ to the positive frequency $\omega(K)=\sqrt{K^{2}+k_{y}^{2}+k_{z}^{2}+m^{2}} \geq m$. After setting $\hat{\mathrm{k}}=\left(\omega, k_{y}, k_{z}\right)$ we get

$$
\begin{aligned}
& A_{\rightarrow}^{\mu}(x, \hat{\mathrm{x}}) \equiv \int_{m}^{\infty} \omega \mathrm{d} \omega \iint_{-\infty}^{\infty} \mathrm{d} k_{y} \mathrm{~d} k_{z} \frac{\theta\left(\hat{\mathrm{k}}^{2}-m^{2}\right)}{\sqrt{\hat{\mathrm{k}}^{2}-m^{2}}} \sum_{r=1}^{3} \mathrm{a}_{\mathbf{k}, r} u_{\mathbf{k}, r}^{\mu}(x, \hat{\mathrm{x}})+\text { H.c. } \\
& =\int_{m}^{\infty} \mathrm{d} \omega \iint_{-\infty}^{\infty} \mathrm{d} k_{y} \mathrm{~d} k_{z} \theta\left(\hat{\mathrm{k}}^{2}-m^{2}\right) \sum_{r=1}^{3}\left[\mathrm{a}_{\hat{\mathrm{k}}, r} u_{\hat{\mathrm{k}}, r}^{\mu}(x, \hat{\mathrm{x}})+\mathrm{a}_{\hat{\mathrm{k}}, r}^{\dagger} u_{\hat{\mathrm{k}}, r}^{\mu *}(x, \hat{\mathrm{x}})\right]
\end{aligned}
$$


where

$$
u_{\hat{\mathrm{k}}, r}^{\mu}(x, \hat{\mathrm{x}})=\frac{\theta(-x) e_{r}^{\mu}(\hat{\mathrm{k}})}{\sqrt{ }\left[(2 \pi)^{3} 2 K(\hat{\mathrm{k}})\right]} \exp \left\{-i \omega t+i y k_{y}+i z k_{z}+i x \sqrt{\hat{\mathrm{k}}^{2}-m^{2}}\right\}
$$

with

$$
e_{r}^{\mu}(\hat{\mathrm{k}})=e_{r}^{\mu}\left(K(\hat{\mathrm{k}}), k_{y}, k_{z}\right) \quad(r=1,2,3) \quad \mathrm{a}_{\mathbf{k}, r} \sqrt{\frac{\omega}{K}}=\mathrm{a}_{\hat{\mathrm{k}}, r}
$$

in such a manner that we come to the canonical commutation relations

$$
\begin{gathered}
{\left[\mathrm{a}_{\hat{\mathrm{k}}, r}, \mathrm{a}_{\hat{\mathrm{k}}^{\prime}, s}\right]=0 \quad\left[\mathrm{a}_{\hat{\mathrm{k}}, r}^{\dagger}, \mathrm{a}_{\hat{\mathrm{k}}^{\prime}, s}^{\dagger}\right]=0} \\
{\left[\mathrm{a}_{\hat{\mathrm{k}}, r}, \mathrm{a}_{\hat{\mathrm{k}}^{\prime}, s}^{\dagger}\right]=\frac{\omega}{K} \delta\left(K-K^{\prime}\right) \delta\left(k_{y}-k_{y}^{\prime}\right) \delta\left(k_{z}-k_{z}^{\prime}\right)=\delta\left(\omega-\omega^{\prime}\right) \delta\left(k_{y}-k_{y}^{\prime}\right) \delta\left(k_{z}-k_{z}^{\prime}\right)}
\end{gathered}
$$

with $\omega, \omega^{\prime} \geq 0$. Notice that the incident or progressive wave functions $u_{\hat{\mathrm{k}}, r}^{\mu}(x, \hat{\mathrm{x}})$ are tempered distributions satisfying the non-homogeneous Klein-Gordon equation

$$
\left(\square+m^{2}\right) u_{\hat{\mathrm{k}}, r}^{\mu}(x, \hat{\mathrm{x}})=-\left\{\delta^{\prime}(x)+i \delta(x) \sqrt{\hat{\mathrm{k}}^{2}-m^{2}}\right\} \frac{e_{r}^{\mu}(\hat{\mathrm{k}})}{\sqrt{ }\left[(2 \pi)^{3} 2 K(\hat{\mathrm{k}})\right]} \exp \{-i \hat{\mathrm{k}} \cdot \hat{\mathrm{x}}\}
$$

By repeating the very same manipulations to the regressive part $A_{\leftarrow}^{\mu}(x, \hat{\mathrm{x}})$ of the Proca-Stückelberg vector field, we eventually come to the normal modes expansion for $x \leq 0$

$$
A_{\leftarrow}^{\nu}(\mathrm{x})=\int_{m}^{\infty} \mathrm{d} \omega \iint_{-\infty}^{\infty} \mathrm{d} k_{y} \mathrm{~d} k_{z} \theta\left(\hat{\mathrm{k}}^{2}-m^{2}\right) \sum_{r=1}^{3}\left[\overline{\mathrm{a}}_{\hat{\mathrm{k}}, r} \bar{u}_{\hat{\mathrm{k}}, r}^{\nu}(\mathrm{x})+\overline{\mathrm{a}}_{\hat{\mathrm{k}}, r}^{\dagger} \bar{u}_{\hat{\mathrm{k}}, r}^{\nu *}(\mathrm{x})\right]
$$

where the reflected or regressive wave functions have the form

$$
\begin{array}{r}
\bar{u}_{\hat{\mathrm{k}}, r}^{\mu}(\mathrm{x})=\theta(-x)\left[(2 \pi)^{3} 2 K(\hat{\mathrm{k}})\right]^{-1 / 2} \bar{e}_{r}^{\mu}(\hat{\mathrm{k}}) \exp \{-i x K(\hat{\mathrm{k}})-i \hat{\mathrm{k}} \cdot \hat{\mathrm{x}}\} \\
\bar{e}_{r}^{\mu}(\hat{\mathrm{k}})=e_{r}^{\mu}\left(-K(\hat{\mathrm{k}}), k_{y}, k_{z}\right) \quad(r=1,2,3)
\end{array}
$$

and do fulfill the non-homogeneous Klein-Gordon equation

$$
\left(\square+m^{2}\right) \bar{u}_{\hat{\mathrm{k}}, r}^{\mu}(x, \hat{\mathrm{x}})=-\left\{\delta^{\prime}(x)-i \delta(x) \sqrt{\hat{\mathrm{k}}^{2}-m^{2}}\right\} \frac{\bar{e}_{r}^{\mu}(\hat{\mathrm{k}})}{\sqrt{ }\left[(2 \pi)^{3} 2 K(\hat{\mathrm{k}})\right]} \exp \{-i \hat{\mathrm{k}} \cdot \hat{\mathrm{x}}\}
$$

while the corresponding creation and annihilation operators satisfy canonical commutation relations

$$
\left[\overline{\mathrm{a}}_{\hat{\mathrm{k}}, r}, \mathrm{a}_{\hat{\mathrm{k}}^{\prime}, s}\right]=\left[\mathrm{a}_{\hat{\mathrm{k}}, r}^{\dagger}, \overline{\mathrm{a}}_{\hat{\mathrm{k}}^{\prime}, s}\right]=0 \quad\left[\overline{\mathrm{a}}_{\hat{\mathrm{k}}, r}, \overline{\mathrm{a}}_{\hat{\mathrm{k}}^{\prime}, s}^{\dagger}\right]=\delta\left(\hat{\mathrm{k}}-\hat{\mathrm{k}}^{\prime}\right) \delta_{r s}
$$

It's worth noticing that all the wave functions are normalized in order to reproduce the constant vector current: for example, for incident or progressive wave functions we find

$$
(2 \pi)^{3} g_{\mu \nu} u_{\hat{\mathrm{k}}, s}^{\mu *}(\mathrm{x}) i \stackrel{\leftrightarrow}{\partial_{\lambda}} u_{\hat{\mathrm{k}}, r}^{\nu}(\mathrm{x})=\delta_{r s} \frac{k_{\lambda}}{K} \quad k^{\mu}=\left(\omega, K=\sqrt{\hat{\mathrm{k}}^{2}-m^{2}}, k_{y}, k_{z}\right)
$$

The normalization of the incident or progressive vector plane waves is such that

$$
\begin{array}{r}
\left.g_{\mu \nu} \int \mathrm{d} \hat{\mathrm{x}} u_{\hat{\mathrm{p}}, r}^{\mu *}(x, \hat{\mathrm{x}}) i \stackrel{\leftrightarrow}{\partial_{\lambda}} u_{\hat{\mathrm{k}}, s}^{\nu}(x, \hat{\mathrm{x}})\right|_{x=0}=\delta(\hat{\mathrm{k}}-\hat{\mathrm{p}}) \delta_{r s} \frac{k_{\lambda}}{K} \\
\left.g_{\mu \nu} \int \mathrm{d} \hat{\mathrm{x}} u_{\hat{\mathrm{p}}, r}^{\mu}(x, \hat{\mathrm{x}}) i \stackrel{\leftrightarrow}{\partial_{\lambda}} u_{\hat{\mathrm{k}}, s}^{\nu *}(x, \hat{\mathrm{x}})\right|_{x=0}=\delta(\hat{\mathrm{k}}-\hat{\mathrm{p}}) \delta_{r s}\left(-\frac{k_{\lambda}}{K}\right) \\
\left.g_{\mu \nu} \int \mathrm{d} \hat{\mathrm{x}} u_{\hat{\mathrm{p}}, r}^{\mu}(x, \hat{\mathrm{x}}) i \stackrel{\leftrightarrow}{\partial_{\lambda}} u_{\hat{\mathrm{k}}, s}^{\nu}(x, \hat{\mathrm{x}})\right|_{x=0}=0
\end{array}
$$


in which $v=\omega / K$ is the (phase) velocity of the vector plane wave $u_{\hat{\mathrm{k}}, r}^{\nu}(\mathrm{x})$. Now, concerning the reflected or regressive plane waves, it is convenient to set

$$
\begin{array}{r}
u_{-\hat{\mathrm{k}}, r}^{\mu}(\mathrm{x})=\theta(-x)\left[(2 \pi)^{3} 2 K(\hat{\mathrm{k}})\right]^{-1 / 2} e_{r}^{\mu}(-\hat{\mathrm{k}}) \exp \{-i x K(\hat{\mathrm{k}})+i \hat{\mathrm{k}} \cdot \hat{\mathrm{x}}\} \\
e_{r}^{\mu}(-\hat{\mathrm{k}})=e_{r}^{\mu}\left(-K(\hat{\mathrm{k}}),-k_{y},-k_{z}\right) \quad(r=1,2,3)
\end{array}
$$

in such a manner that we can recast the normal modes expansion of the Proca-Stückelberg vector field in the simple form

$$
\begin{array}{r}
A_{\mathrm{PS}}^{\mu}(x, \hat{\mathrm{x}})=A_{\rightarrow}^{\mu}(x, \hat{\mathrm{x}})+A_{\leftarrow}^{\mu}(x, \hat{\mathrm{x}})=\int \mathrm{d} \hat{\mathrm{k}} \sum_{r=1}^{3} \mathrm{a}_{\hat{\mathrm{k}}, r} u_{\hat{\mathrm{k}}, r}^{\mu}(x, \hat{\mathrm{x}})+\text { H.c. } \\
\int \mathrm{d} \hat{\mathrm{k}} \equiv \int_{-\infty}^{\infty} \mathrm{d} \omega \theta\left(\omega^{2}-m^{2}\right) \iint_{-\infty}^{\infty} \mathrm{d} k_{y} \mathrm{~d} k_{z} \theta\left(\hat{\mathrm{k}}^{2}-m^{2}\right)
\end{array}
$$

with the canonical commutation relations

$$
\begin{gathered}
{\left[\mathrm{a}_{\hat{\mathrm{k}}, r}, \mathrm{a}_{\hat{\mathrm{k}}^{\prime}, s}\right]=0 \quad\left[\mathrm{a}_{\hat{\mathrm{k}}, r}^{\dagger}, \mathrm{a}_{\hat{\mathrm{k}}^{\prime}, s}^{\dagger}\right]=0} \\
{\left[\mathrm{a}_{\hat{\mathrm{k}}, r}, \mathrm{a}_{\hat{\mathrm{k}}^{\prime}, s}^{\dagger}\right]=\delta\left(\omega-\omega^{\prime}\right) \delta\left(k_{y}-k_{y}^{\prime}\right) \delta\left(k_{z}-k_{z}^{\prime}\right) \quad \omega^{2} \geq m^{2} \vee k_{y}, k_{z} \in \mathbb{R}}
\end{gathered}
$$

On the other hand, the general solutions for $\zeta \cdot x<0$, concerning the Maxwell-Chern-Simons free quantum field, have been extensively discussed and applied in [16, 45, 46] for the massive case and in [47] for the massless case. However, in the light of the present applications, it is better to shortly overview this important topic.

\section{B. Maxwell-Chern-Simons Quantum Field}

In the following we shall review and extend the main tools developed in [48], which are necessary to understand the squeezed pairs emission and absorption in the presence of a parity breaking background medium. Let us first recall the construction of the so called chiral or birefringent polarization vectors for the Maxwell-Chern-Simons (MCS) vector field. Here we aim to develop a rather general frame which could allow for an understanding of the kinematics of massive vector particles in the presence of a space-like Chern-Simons LIV vector. The starting point is the rank-two symmetric matrix [16]

$$
S_{\lambda}^{\nu} \equiv \varepsilon^{\mu \nu \alpha \beta} \zeta_{\alpha} k_{\beta} \varepsilon_{\mu \lambda \rho \sigma} \zeta^{\rho} k^{\sigma}=\delta_{\lambda}^{\nu} \mathrm{D}+k^{\nu} k_{\lambda} \zeta^{2}+\zeta^{\nu} \zeta_{\lambda} k^{2}-\zeta \cdot k\left(\zeta_{\lambda} k^{\nu}+\zeta^{\nu} k_{\lambda}\right)
$$

where

$$
\mathrm{D} \equiv(\zeta \cdot k)^{2}-\zeta^{2} k^{2}=\frac{1}{2} S_{\nu}^{\nu} .
$$

It is convenient to introduce the two orthogonal, one-dimensional, Hermitian projectors

$$
\boldsymbol{\pi}_{ \pm}^{\mu \nu} \equiv \frac{S^{\mu \nu}}{2 \mathrm{D}} \pm \frac{i}{2} \varepsilon^{\mu \nu \alpha \beta} \zeta_{\alpha} k_{\beta} \mathrm{D}^{-\frac{1}{2}}=\left(\boldsymbol{\pi}_{ \pm}^{\nu \mu}\right)^{*}=\left(\boldsymbol{\pi}_{\mp}^{\mu \nu}\right)^{*} \quad(\mathrm{D}>0)
$$

A couple of chiral polarization vectors for the Maxwell-Chern-Simons free vector field can be constructed out of some tetrad of constant quantities $\epsilon_{\nu}$, taking into account that we have

$$
\pi_{ \pm}^{\mu \lambda} \epsilon_{\mu} \epsilon_{\lambda}=\mathrm{D} \epsilon^{2}+\zeta^{2}(\epsilon \cdot k)^{2}=\left[(\zeta \cdot k)^{2}-\zeta^{2} k^{2}\right] \epsilon^{2}+\zeta^{2}(\epsilon \cdot k)^{2}
$$

For example, for the spatial Chern-Simons vector $\zeta^{\mu}=\left(0, \zeta_{x}, 0,0\right)$ we can always build up a pair of space-like, complex, chiral polarization vectors

$$
\varepsilon_{ \pm}^{\mu}(k)=\pi_{ \pm}^{\mu \lambda} \epsilon_{\lambda}\left[\left(k_{y}^{2}-k_{0}^{2}\right) /\left(k_{z}^{2}+k_{y}^{2}-k_{0}^{2}\right)\right]^{-\frac{1}{2}} .
$$

By the very construction, for $\mathrm{D}>0$ this couple of chiral polarization vectors satisfy the conjugation and orthogonality relations

$$
\varepsilon_{ \pm}^{\mu *}(k)=\varepsilon_{\mp}^{\mu}(k) ; \quad-\frac{1}{2} g_{\mu \nu} \varepsilon_{ \pm}^{\mu *}(k) \varepsilon_{ \pm}^{\nu}(k)+\text { c. c. }=1 ; \quad \frac{1}{2} g_{\mu \nu} \varepsilon_{ \pm}^{\mu *}(k) \varepsilon_{\mp}^{\nu}(k)+\text { c. c. }=0
$$


as well as the closure relations

$$
\varepsilon_{+}^{\mu *}(k) \varepsilon_{+}^{\nu}(k)+\varepsilon_{-}^{\mu *}(k) \varepsilon_{-}^{\nu}(k)=\varepsilon_{-}^{\mu}(k) \varepsilon_{+}^{\nu}(k)+\varepsilon_{+}^{\mu}(k) \varepsilon_{-}^{\nu}(k)=\mathrm{D}^{-1} S^{\mu \nu}
$$

In order to obtain the normal modes expansion of the MCS quantum field, let's introduce the kinetic $4 \times 4$ Hermitian matrix $\mathbb{K}$ with complex entries

$$
K_{\lambda \nu} \equiv g_{\lambda \nu}\left(k^{2}-m^{2}\right)+i \varepsilon_{\lambda \nu \alpha \beta} \zeta^{\alpha} k^{\beta}
$$

which satisfy

$$
K_{\lambda \nu}=K_{\nu \lambda}^{*}
$$

Now we are ready to find the general solution of the free field equations (9) for $\zeta \cdot x<0$. From the relationships (40) and (38) we readily obtain

$$
\begin{aligned}
K_{\nu}^{\mu}{ }_{\nu} \varepsilon_{ \pm}^{\nu}(k) & =\left[\delta^{\mu}{ }_{\nu}\left(k^{2}-m^{2}\right)+\sqrt{\mathrm{D}}\left(\boldsymbol{\pi}_{+\nu}^{\mu}-\boldsymbol{\pi}_{-\nu}^{\mu}\right)\right] \varepsilon_{ \pm}^{\mu}(k) \\
& =\left(k^{2}-m^{2} \pm \sqrt{\mathrm{D}}\right) \varepsilon_{ \pm}^{\mu}(k)
\end{aligned}
$$

which shows that the polarization vectors of positive and negative chirality are solutions of the vector field equations for $\zeta \cdot x<0$ iff

$$
\begin{array}{rr}
k_{ \pm}^{\mu}=\left(\omega_{\mathbf{k}, \pm}, \mathbf{k}\right) \quad \varepsilon_{ \pm}^{\mu}(\mathbf{k})=\varepsilon_{ \pm}^{\mu}\left(k_{ \pm}\right) & \left(k_{ \pm}^{0}=\omega_{\mathbf{k}, \pm}\right) \\
\omega_{\mathbf{k}, \pm}=\sqrt{\mathbf{k}^{2}+m^{2}+\frac{1}{2} \zeta_{x}^{2} \pm \zeta_{x} \sqrt{k_{x}^{2}+m^{2}+\frac{1}{4} \zeta_{x}^{2}}} \quad \text { for } \quad \zeta^{\mu}=\left(0, \zeta_{x}, 0,0\right) .
\end{array}
$$

To complete our construction of a basis we introduce the further pair of orthogonal and suitably normalized polarization vectors, respectively the so called scalar and longitudinal polarization real vectors

$$
\begin{aligned}
& \varepsilon_{S}^{\mu}(k) \equiv \frac{k^{\mu}}{\sqrt{k^{2}}} \quad\left(k^{2}>0\right) \\
& \varepsilon_{L}^{\mu}(k) \equiv\left(\mathrm{D} k^{2}\right)^{-\frac{1}{2}}\left(k^{2} \zeta^{\mu}-k^{\mu} \zeta \cdot k\right) \quad\left(k^{2}>0 \vee \mathrm{D}>0\right)
\end{aligned}
$$

which fulfill by construction

$$
\begin{array}{rr}
k_{\mu} \varepsilon_{L}^{\mu}(k)=0 & k_{\mu} \varepsilon_{S}^{\mu}(k)=\sqrt{k^{2}} \quad\left(k^{2}>0\right) \\
g_{\mu \nu} \varepsilon_{S}^{\mu}(k) \varepsilon_{S}^{\nu}(k)=1 \quad g_{\mu \nu} \varepsilon_{L}^{\mu}(k) \varepsilon_{L}^{\nu}(k)=-1 \\
g_{\mu \nu} \varepsilon_{S}^{\mu}(k) \varepsilon_{L}^{\nu}(k)=g_{\mu \nu} \varepsilon_{S}^{\mu}(k) \varepsilon_{ \pm}^{\nu}(k)=g_{\mu \nu} \varepsilon_{L}^{\mu}(k) \varepsilon_{ \pm}^{\nu}(k)=0
\end{array}
$$

Hence we have at our disposal $\forall k^{\mu}$ with $k^{2}>0 \vee \mathrm{D}>0$ a complete and orthogonal chiral set of four polarization vectors: namely,

$$
\varepsilon_{A}^{\mu}(k)=\left\{\begin{array}{cl}
k^{\mu} / \sqrt{k^{2}} & \text { for } A=S \\
\left(k^{2} \zeta^{\mu}-k^{\mu} \zeta \cdot k\right) / \sqrt{\mathrm{D} k^{2}} & \text { for } A=L \\
\varepsilon_{ \pm}^{\mu}\left(k_{ \pm}\right) & \text {for } A= \pm
\end{array} \quad-\left(k^{2}>0 \vee \mathrm{D}>0\right)\right.
$$

In order to fully implement the canonical quantum theory of the MCS massive vector field for the simple choice $\varkappa=1$, it is convenient to introduce the polarized plane waves according to

$$
v_{\mathbf{k} A}^{\nu}(x)=\left[(2 \pi)^{3} 2 \omega_{\mathbf{k} A}\right]^{-\frac{1}{2}} \varepsilon_{A}^{\nu}(k) \exp \left\{-i \omega_{\mathbf{k} A} t+i \mathbf{k} \cdot \mathbf{x}\right\}
$$

where the dispersion relation for the scalar and longitudinal frequencies is the covariant one, viz.,

$$
\omega_{\mathbf{k} S}=\omega_{\mathbf{k} L}=\sqrt{\mathbf{k}^{2}+m^{2}} \equiv \omega_{\mathbf{k}}
$$

so that we can write

$$
k_{\nu} \varepsilon_{S}^{\nu}(k)=m \quad i \partial_{\nu} v_{\mathbf{k} S}^{\nu}(x)=u_{\mathbf{k}}(x)
$$


It follows therefrom that the general solution of the Euler-Lagrange field equations (8) for the quantum massive vector field when $\varkappa=1$ and $\zeta \cdot x<0$ takes the form

$$
\begin{aligned}
A^{\nu}(x) & =A_{\mathrm{CS}}^{\nu}(x)-\partial^{\nu} B(x) / m^{2} \\
A_{\mathrm{CS}}^{\nu}(x) & =\int \mathrm{d} \mathbf{k} \sum_{A= \pm, L}\left[c_{\mathbf{k}, A} v_{\mathbf{k} A}^{\nu}(x)+c_{\mathbf{k}, A}^{\dagger} v_{\mathbf{k} A}^{\nu *}(x)\right] \\
B(x) & =m \int \mathrm{d} \mathbf{k}\left[b_{\mathbf{k}} u_{\mathbf{k}}(x)+b_{\mathbf{k}}^{\dagger} u_{\mathbf{k}}^{*}(x)\right]
\end{aligned}
$$

where the canonical commutation relations holds true, viz.,

$$
\left[c_{\mathbf{k}, A}, c_{\mathbf{k}^{\prime}, A^{\prime}}^{\dagger}\right]=-g_{A A^{\prime}} \delta\left(\mathbf{k}-\mathbf{k}^{\prime}\right) \quad c_{\mathbf{k}, S}=b_{\mathbf{k}}
$$

all the other commutators being equal to zero. According to equations (8) and (9) we obtain

$$
\begin{aligned}
B(x) & =-i \int \mathrm{d} \mathbf{k} k_{\nu}\left[c_{\mathbf{k} S} v_{\mathbf{k} S}^{\nu}(x)-c_{\mathbf{k} S}^{\dagger} v_{\mathbf{k} S}^{\nu *}(x)\right]_{k_{0}=\omega_{\mathbf{k}}} \\
& =m \int \mathrm{d} \mathbf{k}\left[b_{\mathbf{k}} u_{\mathbf{k}}(x)+b_{\mathbf{k}}^{\dagger} u_{\mathbf{k}}^{*}(x)\right]_{k_{0}=\omega_{\mathbf{k}}}
\end{aligned}
$$

in such a manner that the physical Hilbert space $\mathfrak{H}_{\text {phys }}$, with a positive semi-definite metric for all the MCS massive quantum states, is selected out from the Fock space $\mathfrak{F}$ by means of the customary subsidiary condition

$$
\left.\left.B^{(-)}(x) \mid \text { phys }\right\rangle=0 \quad \forall \mid \text { phys }\right\rangle \in \mathfrak{H}_{\text {phys }} \subset \mathfrak{F}
$$

On the other side, the physical MCS massive quanta are created out of the Fock vacuum by the creation part of the quantum physical massive MCS vector field $A_{\mathrm{CS}}^{\nu}(x)$, with the standard non-vanishing commutation relations

$$
\left[c_{\mathbf{k}, A}, c_{\mathbf{k}^{\prime}, A^{\prime}}^{\dagger}\right]=\delta_{A A^{\prime}} \delta\left(\mathbf{k}-\mathbf{k}^{\prime}\right) \quad A, A^{\prime}=L, \pm
$$

all the other commutators being equal to zero. Notice that the MCS massive 1-particle states of definite spatial momentum $\mathbf{k}$ do exhibit three polarization states, i.e., one linear longitudinal polarization of real vector $\varepsilon_{L}^{\nu}(k)$ with dispersion relation $k^{2}=m^{2}$ and two chiral transverse states with complex vectors $\varepsilon_{ \pm}^{\nu}\left(k_{ \pm}\right)$and dispersion relations (43), the negative chirality states $\varepsilon_{-}^{\nu}\left(k_{-}\right)$being well defined only for $\left|\zeta_{\lambda} \cdot k_{-}^{\lambda}\right|<m / \zeta \Leftrightarrow k_{-}^{2}>0$.

\section{THE BOGOLYUBOV TRANSFORMATIONS}

An equivalent rearrangement can be pursued in the right region $\mathrm{R}$, where the parity breaking medium is present. As a result we shall obtain the following normal modes expansion of the Chern-Simons vector field for $x \geq 0$ : namely,

$$
A_{\mathrm{CS}}^{\nu}(\mathrm{x})=\int \mathrm{d} \hat{\mathrm{k}} \sum_{A= \pm, L}\left[\mathrm{c}_{\hat{\mathrm{k}}, A} v_{\hat{\mathrm{k}}, A}^{\nu}(\mathrm{x})+\mathrm{c}_{\hat{\mathrm{k}}, A}^{\dagger} v_{\hat{\mathrm{k}}, A}^{\nu *}(\mathrm{x})\right]
$$

in which, in full analogy with the Proca-Stückelberg plane wave solution (22), the incident or progressive plane wave solution for the Chern-Simons classical field equations is provided by

$$
\begin{array}{r}
v_{\hat{\mathrm{k}} A}^{\nu}(\mathrm{x})=\theta(x)\left[(2 \pi)^{3} 2 K_{A}\right]^{-\frac{1}{2}} \varepsilon_{A}^{\nu}(\hat{\mathrm{k}}) \exp \left\{i x K_{A}(\hat{\mathrm{k}})-i \hat{\mathrm{k}} \cdot \hat{\mathrm{x}}\right\} \\
K_{A}=\left\{\begin{array}{cc}
\sqrt{\hat{\mathrm{k}}^{2}-m^{2} \pm \sqrt{\zeta_{x}^{2} \hat{\mathrm{k}}^{2}}} & \text { for } A= \pm \\
K=\sqrt{\hat{\mathrm{k}}^{2}-m^{2}} & \text { for } A=L
\end{array}\right.
\end{array}
$$

while the canonical commutation relations still hold true, viz.,

$$
\left[\mathrm{c}_{\hat{\mathrm{k}}, A}, \mathrm{c}_{\hat{\mathrm{k}}^{\prime}, B}\right]=\left[\mathrm{c}_{\hat{\mathrm{k}}, A}^{\dagger}, \mathrm{c}_{\hat{\mathrm{k}}^{\prime}, B}^{\dagger}\right]=0 \quad\left[\mathrm{c}_{\hat{\mathrm{k}}, A}, \mathrm{c}_{\hat{\mathrm{k}}^{\prime}, B}^{\dagger}\right]=\delta\left(\hat{\mathrm{k}}-\hat{\mathrm{k}}^{\prime}\right) \delta_{A B}
$$


Coming back to the boundary conditions, it turns out that the matching condition for the vector fields at the hyperplane $x=0$ does take the form

$$
\begin{aligned}
& \int \mathrm{d} \hat{\mathrm{k}}\left\{\sum_{A}\left[\mathrm{c}_{\hat{\mathrm{k}}, A} v_{\hat{\mathrm{k}}, A}^{\mu}(\hat{\mathrm{x}})+\mathrm{c}_{\hat{\mathrm{k}}, A}^{\dagger} v_{\hat{\mathrm{k}}, A}^{\mu *}(\hat{\mathrm{x}})\right]\right. \\
& \left.-\sum_{r=1}^{3}\left[\mathrm{a}_{\hat{\mathrm{k}}, r} u_{\hat{\mathrm{k}}, r}^{\mu}(\hat{\mathrm{x}})+\mathrm{a}_{\hat{\mathrm{k}}, r}^{\dagger} u_{\hat{\mathrm{k}}, r}^{\mu *}(\hat{\mathrm{x}})\right]\right\}=0
\end{aligned}
$$

which can be implemented if we perform a Bogolyubov transformation

$$
v_{\hat{\mathrm{k}}, A}^{\nu}(\hat{\mathrm{x}})=\sum_{s=1}^{3}\left[\alpha_{s A}(\hat{\mathrm{k}}) u_{\hat{\mathrm{k}}, s}^{\nu}(\hat{x})-\beta_{s A}(\hat{\mathrm{k}}) u_{\hat{\mathrm{k}}, s}^{\nu *}(\hat{\mathrm{x}})\right]
$$

where the complex numerical coefficients $\alpha_{s A}(\hat{\mathrm{k}}), \beta_{s A}(\hat{\mathrm{k}})$ will be determined below. On one hand we find

$$
\begin{aligned}
\left\langle u_{\hat{\mathrm{p}}, r}^{\mu} \mid v_{\hat{\mathrm{k}}, A}^{\nu}\right\rangle & \left.\equiv \int \mathrm{d} \hat{\mathrm{x}} u_{\hat{\mathrm{p}}, r}^{\mu *}(x, \hat{\mathrm{x}})(-i) \overleftrightarrow{\partial_{x}} v_{\hat{\mathrm{k}}, A}^{\nu}(x, \hat{\mathrm{x}})\right|_{x=0} \\
& =\delta(\hat{\mathrm{k}}-\hat{\mathrm{p}}) e_{r}^{\mu}(\hat{\mathrm{k}}) \frac{K_{A}+K}{2 \sqrt{ }\left(K_{A} K\right)} \varepsilon_{A}^{\nu}(\hat{\mathrm{k}})
\end{aligned}
$$

and on the other hand we evidently obtain

$$
\left\langle u_{\hat{\mathrm{p}}, r}^{\mu} \mid u_{\hat{\mathrm{k}}, s}^{\lambda}\right\rangle=\delta(\hat{\mathrm{k}}-\hat{\mathrm{p}}) e_{r}^{\mu}(\hat{\mathrm{k}}) e_{s}^{\lambda}(\hat{\mathrm{k}})=-\left\langle u_{\hat{\mathrm{p}}, r}^{\mu *} \mid u_{\hat{\mathrm{k}}, s}^{\lambda *}\right\rangle ;\left\langle u_{\hat{\mathrm{p}}, r}^{\mu} \mid u_{\hat{\mathrm{k}}, s}^{\lambda *}\right\rangle=0
$$

and thereby

$$
\begin{aligned}
\left\langle u_{\hat{\mathrm{p}}, r}^{\mu} \mid v_{\hat{\mathrm{k}}, A}^{\nu}\right\rangle & =\delta(\hat{\mathrm{k}}-\hat{\mathrm{p}}) e_{r}^{\mu}(\hat{\mathrm{k}}) \sum_{s=1}^{3} \alpha_{s A}(\hat{\mathrm{k}}) e_{s}^{\nu}(\hat{\mathrm{k}}) \\
\left\langle u_{\hat{\mathrm{p}}, r}^{\mu *} \mid v_{\hat{\mathrm{k}}, A}^{\nu}\right\rangle & =\delta(\hat{\mathrm{k}}-\hat{\mathrm{p}}) e_{r}^{\mu}(\hat{\mathrm{k}}) \sum_{s=1}^{3} \beta_{s A}(\hat{\mathrm{k}}) e_{s}^{\nu}(\hat{\mathrm{k}})
\end{aligned}
$$

A comparison yields - by omitting the argument $\hat{\mathrm{k}}$

$$
\frac{K_{A}+K}{2 \sqrt{ }\left(K_{A} K\right)} \varepsilon_{A}^{\nu}=\sum_{s=1}^{3} \alpha_{s A} e_{s}^{\nu} \quad \frac{K_{A}-K}{2 \sqrt{ }\left(K_{A} K\right)} \varepsilon_{A}^{\nu}=\sum_{s=1}^{3} \beta_{s A} e_{s}^{\nu}
$$

the solution of which is provided by

$$
\alpha_{s A}=\frac{1}{2} e_{s} \cdot \varepsilon_{A} \frac{K_{A}+K}{\sqrt{ }\left(K_{A} K\right)} \quad \beta_{s A}=\frac{1}{2} e_{s} \cdot \varepsilon_{A} \frac{K_{A}-K}{\sqrt{ }\left(K_{A} K\right)}
$$

as it can be readily checked by direct inspection, where the wave vectors $K, K_{A}$ are positive functions of $\hat{\mathrm{k}}$ as given by eq.s (61). Let's evaluate the quantity

$$
\sum_{s=1}^{3}\left[\alpha_{s A}(\hat{k}) \alpha_{s B}^{*}(\hat{k})-\beta_{s A}(\hat{k}) \beta_{s B}^{*}(\hat{k})\right] \quad \text { for } \quad A, B=L, \pm
$$

First we find

$$
\begin{aligned}
& \frac{1}{2} g_{\mu \nu} \varepsilon_{A}^{\mu}(\hat{\mathrm{k}}) \frac{1}{2} g_{\rho \sigma} \varepsilon_{B}^{\rho *}(\hat{\mathrm{k}}) \sum_{s=1}^{3} e_{s}^{\nu}(\hat{\mathrm{k}}) e_{s}^{\sigma}(\hat{\mathrm{k}}) \\
= & \frac{1}{4} \varepsilon_{A}^{\mu}(\hat{\mathrm{k}}) \varepsilon_{B}^{\rho *}(\hat{\mathrm{k}})\left(-g_{\rho \mu}+\frac{k_{\rho} k_{\mu}}{m^{2}}\right)=-\frac{1}{4} g_{\rho \mu} \varepsilon_{A}^{\mu}(\hat{\mathrm{k}}) \varepsilon_{B}^{\rho *}(\hat{\mathrm{k}})=\frac{1}{4} \delta_{A B}
\end{aligned}
$$


where use has been made of the transverse-like condition, as well as the orthogonality relations, together with the fact that the covariant linear polarization vectors have been chosen to be real. Hence we eventually obtain

$$
\begin{aligned}
& \sum_{s=1}^{3} \alpha_{s A}(\hat{\mathrm{k}}) \alpha_{s B}^{*}(\hat{\mathrm{k}})=\delta_{A B} \frac{\left(K_{A}+K\right)^{2}}{4 K_{A} K} \\
& \sum_{s=1}^{3} \beta_{s A}(\hat{\mathrm{k}}) \beta_{s B}^{*}(\hat{\mathrm{k}})=\delta_{A B} \frac{\left(K_{A}-K\right)^{2}}{4 K_{A} K}
\end{aligned}
$$

Subtraction of the above expressions yields the customary relation

$$
\sum_{s=1}^{3}\left[\alpha_{s A}(\hat{\mathrm{k}}) \alpha_{s B}^{*}(\hat{\mathrm{k}})-\beta_{s A}(\hat{\mathrm{k}}) \beta_{s B}^{*}(\hat{\mathrm{k}})\right]=\delta_{A B} \quad[A, B=L, \pm]
$$

and by making quite analogous manipulations one can readily check that the further Bogolyubov relations

$$
\begin{array}{ll}
\sum_{s=1}^{3}\left[\alpha_{s A}(\hat{\mathrm{k}}) \beta_{s B}^{*}(\hat{\mathrm{k}})-\beta_{s A}(\hat{\mathrm{k}}) \alpha_{s B}^{*}(\hat{\mathrm{k}})\right]=0 & {[A, B=L, \pm]} \\
\sum_{s=1}^{3}\left[\alpha_{s A}(\hat{\mathrm{k}}) \beta_{s B}(\hat{\mathrm{k}})-\beta_{s B}(\hat{\mathrm{k}}) \alpha_{s A}(\hat{\mathrm{k}})\right]=0 & {[A, B=L, \pm]}
\end{array}
$$

Turning back to the boundary condition (64) and taking the Bogolyubov transformation (65) into account, we can write the operator equality

$$
\begin{array}{r}
\mathrm{a}_{\hat{\mathrm{k}}, r}=\sum_{A= \pm, L}\left[\alpha_{r A}(\hat{\mathrm{k}}) \mathrm{c}_{\hat{\mathrm{k}}, A}-\beta_{r A}^{*}(\hat{\mathrm{k}}) \mathrm{c}_{\hat{\mathrm{k}}, A}^{\dagger}\right] \\
\mathrm{c}_{\hat{\mathrm{k}}, A}=\sum_{r=1}^{3}\left[\alpha_{A r}^{*}(\hat{\mathrm{k}}) \mathrm{a}_{\hat{\mathrm{k}}, r}+\beta_{A r}^{*}(\hat{\mathrm{k}}) \mathrm{a}_{\hat{\mathrm{k}}, r}^{\dagger}\right]
\end{array}
$$

From the canonical commutation relations (62) we obtain

$$
\begin{aligned}
{\left[\mathrm{a}_{\hat{\mathrm{k}}, r}, \mathrm{a}_{\hat{\mathrm{p}}, s}^{\dagger}\right] } & =\delta(\hat{\mathrm{k}}-\hat{\mathrm{p}}) \delta_{r s} \\
& =\sum_{A, B= \pm, L}\left[\alpha_{r A}(\hat{\mathrm{k}}) \mathrm{c}_{\hat{\mathrm{k}}, A}-\beta_{r A}^{*}(\hat{\mathrm{k}}) \mathrm{c}_{\hat{\mathrm{k}}, A}^{\dagger}, \alpha_{s B}^{*}(\hat{\mathrm{p}}) \mathrm{c}_{\hat{\mathrm{p}}, B}^{\dagger}-\beta_{s B}(\hat{\mathrm{p}}) \mathrm{c}_{\hat{\mathrm{p}}, B}\right] \\
& =\sum_{A= \pm, L}\left[\alpha_{r A}(\hat{\mathrm{k}}) \alpha_{s A}^{*}(\hat{\mathrm{p}})-\beta_{r A}(\hat{\mathrm{k}}) \beta_{s A}^{*}(\hat{\mathrm{p}})\right] \delta(\hat{\mathrm{k}}-\hat{\mathrm{p}})
\end{aligned}
$$

and consequently

$$
\sum_{A= \pm, L}\left[\alpha_{r A}(\hat{\mathrm{k}}) \alpha_{s A}^{*}(\hat{\mathrm{k}})-\beta_{r A}(\hat{\mathrm{k}}) \beta_{s A}^{*}(\hat{\mathrm{k}})\right]=\delta_{r s}
$$

Finally, the null commutators $\left[\mathrm{a}_{\hat{\mathrm{k}}, r}, \mathrm{a}_{\hat{\mathrm{p}}, s}\right]=\left[\mathrm{a}_{\hat{\mathrm{k}}, r}^{\dagger}, \mathrm{a}_{\hat{\mathrm{p}}, s}^{\dagger}\right]=0$ lead to the further relations

$$
\begin{aligned}
& \sum_{A= \pm, L}\left[\alpha_{r A}(\hat{\mathrm{k}}) \beta_{s A}^{*}(\hat{\mathrm{k}})-\beta_{r A}(\hat{\mathrm{k}}) \alpha_{s A}^{*}(\hat{\mathrm{k}})\right]=0 \\
& \sum_{A= \pm, L}\left[\alpha_{r A}(\hat{\mathrm{k}}) \beta_{s A}(\hat{\mathrm{k}})-\beta_{r A}(\hat{\mathrm{k}}) \alpha_{s A}(\hat{\mathrm{k}})\right]=0
\end{aligned}
$$

There are two different Fock vacuum states: namely,

$$
\mathrm{a}_{\hat{\mathrm{k}}, r}|0\rangle=0 \quad \mathrm{c}_{\hat{\mathrm{k}}, A}|\Omega\rangle=0
$$


whence from (76)

$$
\mathrm{c}_{\hat{\mathrm{k}}, A}|0\rangle=\sum_{r=1}^{3} \beta_{A r}^{*}(\hat{\mathrm{k}}) \mathrm{a}_{\hat{\mathrm{k},}, r}^{\dagger}|0\rangle
$$

and consequently

$$
\begin{aligned}
\left\langle 0\left|\mathrm{c}_{\hat{\mathrm{p}}, B}^{\dagger} \mathrm{c}_{\hat{\mathrm{k}}, A}\right| 0\right\rangle & =\sum_{r, s=1}^{3}\left\langle 0\left|\mathrm{a}_{\hat{\mathrm{p}}, s} \mathrm{a}_{\hat{\mathrm{k}}, r}^{\dagger}\right| 0\right\rangle \beta_{B s}(\hat{\mathrm{p}}) \beta_{A r}^{*}(\hat{\mathrm{k}}) \\
& =\delta(\hat{\mathrm{k}}-\hat{\mathrm{p}}) \sum_{r=1}^{3} \beta_{B r}(\hat{\mathrm{k}}) \beta_{A r}^{*}(\hat{\mathrm{k}})=\delta(\hat{\mathrm{k}}-\hat{\mathrm{p}}) \delta_{A B} \frac{\left(K_{A}-K\right)^{2}}{4 K_{A} K}
\end{aligned}
$$

In turn we evidently obtain

$$
\left[\mathrm{a}_{\hat{\mathrm{k}}, r}+\sum_{A=L, \pm} \beta_{r A}^{*}(\hat{\mathrm{k}}) \mathrm{c}_{\hat{\mathrm{k}}, A}^{\dagger}\right]|\Omega\rangle=0
$$

that yields

$$
\begin{aligned}
\left\langle\Omega\left|\mathrm{a}_{\hat{\mathrm{p}}, s}^{\dagger} \mathrm{a}_{\hat{\mathrm{k}}, r}\right| \Omega\right\rangle & =\sum_{A, B=L, \pm}\left\langle\Omega\left|\mathrm{c}_{\hat{\mathrm{p}}, A} \mathrm{c}_{\hat{\mathrm{k}}, B}^{\dagger}\right| \Omega\right\rangle \beta_{B s}(\hat{\mathrm{p}}) \beta_{A r}^{*}(\hat{\mathrm{k}}) \\
& =\delta(\hat{\mathrm{k}}-\hat{\mathrm{p}}) \sum_{A=L, \pm} \beta_{A s}(\hat{\mathrm{k}}) \beta_{A r}^{*}(\hat{\mathrm{k}})
\end{aligned}
$$

Moreover we get

$$
\begin{aligned}
\left\langle 0\left|\mathrm{a}_{\hat{\mathrm{p}}, s} \mathrm{c}_{\hat{\mathrm{k}}, A}\right| 0\right\rangle & =\left\langle 0\left|\mathrm{a}_{\hat{\mathrm{p}}, s} \sum_{r=1}^{3}\left[\alpha_{A r}^{*}(\hat{\mathrm{k}}) \mathrm{a}_{\hat{\mathrm{k}}, r}+\beta_{A r}^{*}(\hat{\mathrm{k}}) \mathrm{a}_{\hat{\mathrm{k}}, r}^{\dagger}\right]\right| 0\right\rangle \\
& =\sum_{r=1}^{3} \beta_{A r}^{*}(\hat{\mathrm{k}})\left\langle 0\left|\mathrm{a}_{\hat{\mathrm{p}}, s} \mathrm{a}_{\hat{\mathrm{k}}, r}^{\dagger}\right| 0\right\rangle=\delta(\hat{\mathrm{k}}-\hat{\mathrm{p}}) \beta_{A s}^{*}(\hat{\mathrm{k}}) \\
\left\langle 0\left|\mathrm{a}_{\hat{\mathrm{p}}, s} \mathrm{c}_{\hat{\mathrm{k}}, A}^{\dagger}\right| 0\right\rangle & =\left\langle 0\left|\mathrm{a}_{\hat{\mathrm{p}}, s} \sum_{r=1}^{3}\left[\alpha_{A r}(\hat{\mathrm{k}}) \mathrm{a}_{\hat{\mathrm{k}}, r}^{\dagger}+\beta_{A r}(\hat{\mathrm{k}}) \mathrm{a}_{\hat{\mathrm{k}}, r}\right]\right| 0\right\rangle \\
& =\sum_{r=1}^{3} \alpha_{A r}(\hat{\mathrm{k}})\left\langle 0\left|\mathrm{a}_{\hat{\mathrm{p}}, s} \mathrm{a}_{\hat{\mathrm{k}}, r}^{\dagger}\right| 0\right\rangle=\delta(\hat{\mathrm{k}}-\hat{\mathrm{p}}) \alpha_{A s}(\hat{\mathrm{k}})
\end{aligned}
$$

The latter quantity $\alpha_{A s}(\hat{\mathrm{k}})$ can thereof be interpreted as the relative probability amplitude that a particle of mass $m$, frequency $\omega$, wave vector $\left(K_{A}>0, k_{y}, k_{z}\right)$ and chiral polarization vector $\varepsilon_{A}^{\mu}(\hat{\mathrm{k}})$ is transmitted from the left face to the right face through the hyper-plane $x=0$ by an incident Proca-Stückelberg particle with equal mass $m$ and frequency $\omega$, wave vector $\left(K>0, k_{y}, k_{z}\right)$ but polarization vector $e_{s}^{\mu}(\hat{\mathrm{k}})$. As an effect of this transmission, the first component of wave vector of a birefringent massive particle changes from $K$ to $K_{ \pm}$, while the longitudinal massive quanta do not change their wave vectors.

\section{THE FUNCTIONAL SQUEEZE OPERATOR ALGEBRA}

In this Section we aim to apply the general method developed in [39] to the present context, in order to calculate the squeezed pairs production in the presence of a parity breaking medium. To start with, consider e.g. the pair annihilation and production squeeze operators for the Chern-Simons vector quanta arising in the presence of a parity breaking background medium: namely,

$$
\begin{aligned}
& \Pi(z) \equiv \sum_{\jmath \in \mathfrak{Q}} \frac{1}{2} z_{\jmath} \mathrm{c}_{\jmath}^{2}=\sum_{\jmath \in \mathfrak{Q}} \frac{1}{2} z_{\jmath} \Pi_{\jmath} \\
& \Pi^{\dagger}(\bar{z}) \equiv \sum_{\imath \in \mathfrak{Q}} \frac{1}{2} \bar{z}_{\imath} \mathrm{c}_{\imath}^{\dagger}{ }^{2}=\sum_{\imath \in \mathfrak{Q}} \frac{1}{2} \bar{z}_{\imath} \Pi_{\imath}^{\dagger}
\end{aligned}
$$


where we use the short notation

$$
\sum_{\imath \in \mathfrak{Q}}=\int \mathrm{d} \hat{\mathrm{k}} \sum_{A= \pm, L} \quad\left(\hat{\mathrm{k}} \in \mathbb{R}^{3}\right)
$$

whereas $z_{\imath} \equiv z_{A}(\hat{\mathrm{k}})$ are complex valued dimensionless functions such that

$$
\mathcal{V} \sum_{\imath \in \mathfrak{Q}} z_{\imath} \bar{z}_{\imath}=\mathcal{V} \int \mathrm{d} \hat{\mathrm{k}} \sum_{A= \pm, L}\left|z_{A}(\hat{\mathrm{k}})\right|^{2}=\nu_{o}
$$

is a pure number that will be named the characteristic number of the given squeezed pairs distribution function $z_{A}(\hat{\mathrm{k}})$, while $\mathcal{V}$ is the volume of a symmetric and cubic box in the $1+2$-dimensional Minkowski space $\mathbb{M}_{1}^{2}$, since we set as it is customary

$$
\lim _{\hat{k} \rightarrow 0} \int \mathrm{d} \hat{x} \exp \{ \pm i \hat{k} \cdot \hat{x}\}=(2 \pi)^{3} \delta(\hat{k}=0)=\lim _{\mathcal{V} \rightarrow \infty} \int_{\mathcal{V}} \mathrm{d} \hat{x} \Longleftrightarrow \delta(0) \doteqdot \frac{\mathcal{V}}{(2 \pi)^{3}}
$$

It is worthwhile to remark that the whole algebraic construction we are going to set up in the presence Section does actually live on the boundary hyper-plane $\zeta \cdot x=0$, as it is apparent from the above introduced notations in the related Fourier space.

The creation and destruction operators for Chern-Simons vector particles $\left(c_{\imath}, c_{j}^{\dagger}\right)$ and Proca-Stueckelberg vector particles $\left(\mathrm{a}_{\imath}, \mathrm{a}_{j}^{\dagger}\right)$ do satisfy e.g. the canonical commutation relations (62)

$$
\left[\mathrm{c}_{\imath}, \mathrm{c}_{\jmath}^{\dagger}\right]=\delta_{\imath \jmath}=\left[\mathrm{a}_{\imath}, \mathrm{a}_{\jmath}^{\dagger}\right] \quad(\imath, \jmath \in \mathfrak{Q})
$$

all the remaining commutators being equal to zero. The Fock vacuum states are defined in accordance with the Bogolyubov transformations (75) and (76), that means

$$
\begin{aligned}
& \mathrm{a}_{\hat{\mathrm{k}}, r}|0\rangle=0 \Longleftrightarrow \sum_{A= \pm, L} \alpha_{r A}(\hat{\mathrm{k}}) \mathrm{c}_{\hat{\mathrm{k}}, A}|0\rangle=\sum_{A= \pm, L} \beta_{r A}^{*}(\hat{\mathrm{k}})|\hat{\mathrm{k}} A\rangle \\
& \left(\forall r=1,2,3 \vee \hat{\mathrm{k}} \in \mathbb{R}^{3}\right) \\
& \mathrm{c}_{\hat{\mathrm{k}}, A}|\Omega\rangle=0 \Longleftrightarrow \sum_{r=1}^{3}\left[\alpha_{A r}^{*}(\hat{\mathrm{k}}) \mathrm{a}_{\hat{\mathrm{k}}, r}+\beta_{A r}^{*}(\hat{\mathrm{k}}) \mathrm{a}_{\hat{\mathrm{k}}, r}^{\dagger}\right]|\Omega\rangle=0 \\
& \left(\forall A= \pm, L \vee \hat{\mathrm{k}} \in \mathbb{R}^{3}\right)
\end{aligned}
$$

If we denote by $Q_{a}(a=1,2, \ldots, n)$ any of the $n$ conserved charges of the system, which are allowed by the parity breaking background field configuration, i.e.

$$
Q_{a}=\sum_{\jmath \in \mathfrak{Q}} q_{a \jmath} \mathrm{c}_{\jmath}^{\dagger} \mathrm{c}_{\jmath} \quad(a=1,2, \ldots, n)
$$

where e.g. $q_{a \jmath}(a=1,2, \ldots, n)$ are the charges of the squeezed state with definite quantum numbers $\jmath \in \mathfrak{Q}$, then for any squeezed state $\Pi_{\imath}^{\dagger}|0\rangle$ of definite quantum numbers we evidently find

$$
Q_{a} \Pi_{\imath}^{\dagger}|\Omega\rangle=2 q_{a \imath} \Pi_{\imath}^{\dagger}|\Omega\rangle \quad(\forall \imath \in \mathfrak{Q} \vee a=1,2, \ldots, n)
$$

The creation and annihilation squeeze operators satisfy the commutation relations

$$
\left[\Pi(z), \Pi^{\dagger}(\bar{z})\right]=\sum_{\imath \in \mathfrak{Q}} \frac{1}{2} z_{\imath} \bar{z}_{\imath}\left\{\mathrm{c}_{\imath}, \mathrm{c}_{\imath}^{\dagger}\right\} \equiv 2 \mathrm{~N}(\bar{z} z)
$$

in which

$$
\mathrm{N}(\bar{z} z)=\mathrm{N}^{\dagger}(z \bar{z})=\frac{1}{4} \nu_{o}+\sum_{\imath \in \mathfrak{Q}} \frac{1}{2} z_{\imath} \bar{z}_{\imath} \mathrm{c}_{\imath}^{\dagger} \mathrm{c}_{\imath}
$$


Finally for $\nu_{\imath} \in \mathbb{R}(\forall \imath \in \mathfrak{Q})$ we get

$$
\begin{aligned}
{[\mathrm{N}(\nu), \Pi(z)] } & =-\sum_{\imath \in \mathfrak{Q}} \frac{1}{2} \nu_{\imath} z_{\imath} \Pi_{\imath}=-\Pi(z \nu) \\
{\left[\mathrm{N}(\nu), \Pi^{\dagger}(\bar{z})\right] } & =\sum_{\imath \in \mathfrak{Q}} \frac{1}{2} \nu_{\imath} \bar{z}_{\imath} \Pi_{\imath}^{\dagger}=\Pi^{\dagger}(\nu \bar{z})
\end{aligned}
$$

It follows therefrom that the above three operators do satisfy the well known commutation relations

$$
\begin{aligned}
& {\left[\mathrm{N}(\nu), \Pi^{\dagger}(\bar{z})\right]=\Pi^{\dagger}(\nu \bar{z})} \\
& {[\Pi(z), \mathrm{N}(\nu)]=\Pi(\nu z)} \\
& {\left[\Pi^{\dagger}(\bar{z}), \Pi(z)\right]=2 \mathrm{~N}(\bar{z} z)}
\end{aligned}
$$

in which

$$
\begin{aligned}
\Pi(z)=J_{-}(z)= & J_{1}(z)-i J_{2}(z) \\
\Pi^{\dagger}(\bar{z})=J_{+}(\bar{z})= & J_{1}(\bar{z})+i J_{2}(\bar{z}) \\
& \mathrm{N}(\nu)=J_{3}(z \bar{z})
\end{aligned}
$$

where the threesome of operators

$$
\begin{array}{r}
J_{1}(z, \bar{z}) \equiv \frac{1}{2}\left(\Pi(z)+\Pi^{\dagger}(\bar{z})\right) \\
J_{2}(z, \bar{z}) \equiv \frac{1}{2 i}\left(\Pi^{\dagger}(\bar{z})-\Pi(z)\right) \\
J_{3}(z \bar{z})=\mathrm{N}(\nu)
\end{array}
$$

are a basis of Hermitian generators obeying the well known SU(2) Lie algebra

$$
\left[J_{a}, J_{b}\right]=i \varepsilon_{a b c} J_{c} \quad(a, b, c=1,2,3)
$$

Now it turns out that the quantum state $|\bar{z}\rangle$, which represents a generic squeezed pair of Chern-Simons particles with a momentum distribution function $\bar{z}_{\imath}(\iota \in \mathfrak{Q})$, does satisfy

$$
|\bar{z}\rangle=\Pi^{\dagger}(\bar{z})|\Omega\rangle \quad\langle z|=\langle\Omega| \Pi(z)
$$

and exhibits the infrared regularized normalization

$$
\langle z \mid \bar{z}\rangle=\left\langle\Omega\left|\left[\Pi(z), \Pi^{\dagger}(\bar{z})\right]\right| \Omega\right\rangle=-2\langle\Omega|\mathrm{N}(\bar{z} z)| \Omega\rangle=\nu_{o}
$$

\section{A. Transmission and Reflection from the Algebraic Approach}

The general feature that characterizes the squeezed pairs production and annihilation processes of massive vector particles in the presence of a parity breaking medium is the existence of a non-singular Bogolyubov similarity transformation $\mathcal{S}$, the generator of which is acting on the Fock space according to

$$
\begin{aligned}
\mathrm{a}_{\imath} & =\mathcal{S}^{-1} \mathrm{c}_{\imath} \mathcal{S} \equiv \sum_{\jmath \in \mathfrak{Q}}\left(\alpha_{\imath \jmath} \mathrm{c}_{\jmath}-\beta_{\imath \jmath}^{*} \mathrm{c}_{\jmath}^{\dagger}\right) \\
\mathrm{c}_{\imath}^{\dagger}=\mathcal{S}^{-1} \mathrm{a}_{\imath}^{\dagger} \mathcal{S} & \equiv \sum_{\jmath \in \mathfrak{Q}}\left(\alpha_{\imath \jmath} \mathrm{a}_{\jmath}^{\dagger}+\beta_{\imath \jmath} \mathrm{a}_{\jmath}\right)
\end{aligned}
$$

in agreement with the previous relationships (75) and (76), where

$$
\begin{gathered}
\alpha_{\imath \jmath} \equiv \alpha_{r A}(\hat{\mathrm{k}}, \hat{\mathrm{p}})=\alpha_{r A}(\hat{\mathrm{k}}) \delta(\hat{\mathrm{k}}-\hat{\mathrm{p}})(2 \pi)^{3} \\
\beta_{\imath \jmath} \equiv \beta_{r A}(\hat{\mathrm{k}}, \hat{\mathrm{p}})=\beta_{r A}(\hat{\mathrm{k}}) \delta(\hat{\mathrm{k}}-\hat{\mathrm{p}})(2 \pi)^{3} \\
\alpha_{r A}(\hat{\mathrm{k}})=\frac{1}{2} e_{r}(\hat{\mathrm{k}}) \cdot \varepsilon_{A}(\hat{\mathrm{k}}) \frac{K_{A}+K}{\sqrt{ }\left(K_{A} K\right)} \\
\beta_{r A}(\hat{\mathrm{k}})=\frac{1}{2} e_{r}(\hat{\mathrm{k}}) \cdot \varepsilon_{A}(\hat{\mathrm{k}}) \frac{K_{A}-K}{\sqrt{ }\left(K_{A} K\right)}
\end{gathered}
$$


together with

$$
\sum_{\imath \in \mathfrak{Q}}\left\{\begin{array}{c}
\alpha_{\imath \jmath}^{*} \alpha_{\imath \varkappa}-\beta_{\imath \jmath}^{*} \beta_{\imath \varkappa}=\delta_{\jmath \varkappa} \\
\alpha_{\imath \jmath} \beta_{\imath \varkappa}^{*}-\alpha_{\imath \jmath}^{*} \beta_{\imath \varkappa}=0
\end{array} \quad(\forall \imath, \jmath, \varkappa \in \mathfrak{Q})\right.
$$

in such a manner that the canonical commutation relations (62)

$$
\left[\mathrm{c}_{\imath}, \mathrm{c}_{j}^{\dagger}\right]=\delta_{\imath \jmath}=\left[\mathrm{a}_{\imath}, \mathrm{a}_{j}^{\dagger}\right] \quad \text { et cetera }
$$

keep unchanged thanks to the similarity nature of the non-singular transformation $\mathcal{S}$. Notice that in the present framework the Bogolyubov coefficients $\alpha_{\imath \jmath}$ and $\beta_{\imath \jmath}$ can always be chosen to be real - see eq. (71) for linear polarization.

It follows that we come to the two Fock spaces $\mathfrak{F}_{\text {PS }}$ and $\mathfrak{F}_{\text {CS }}$ which are generated by the cyclic vacuum states normalized to one and defined by

$$
\begin{array}{cc}
\mathrm{a}_{\imath}|0\rangle=0=\langle 0| \mathrm{a}_{\imath}^{\dagger} & (\forall \imath \in \mathfrak{Q}) \\
\mathrm{c}_{\jmath}|\Omega\rangle=0=\langle\Omega| \mathrm{c}_{\jmath}^{\dagger} & (\forall \jmath \in \mathfrak{Q})
\end{array}
$$

Now we have, for example,

$$
\begin{aligned}
\mathrm{c}_{\imath} \mathrm{a}_{\varkappa}^{\dagger}|0\rangle & =\sum_{\jmath \in \mathfrak{Q}}\left(\alpha_{\imath \jmath}^{*} \mathrm{a}_{\jmath} \mathrm{a}_{\varkappa}^{\dagger}|0\rangle+\beta_{\imath \jmath}^{*} \mathrm{a}_{\jmath}^{\dagger} \mathrm{a}_{\varkappa}^{\dagger}|0\rangle\right) \\
& =\alpha_{\imath \varkappa}^{*}|0\rangle+\sum_{\jmath \in \mathfrak{Q}} \beta_{\imath \jmath}^{*} \mathrm{a}_{\jmath}^{\dagger} \mathrm{a}_{\varkappa}^{\dagger}|0\rangle
\end{aligned}
$$

so that

$$
\left\langle 0\left|\mathrm{c}_{\imath} \mathrm{a}_{\jmath}^{\dagger}\right| 0\right\rangle=\alpha_{\imath \jmath}^{*} \quad(\forall \imath, \jmath \in \mathfrak{Q})
$$

In a quite analogous way we get

$$
\begin{aligned}
\mathrm{a}_{\imath} \mathrm{c}_{\varkappa}^{\dagger}|\Omega\rangle & =\sum_{\jmath \in \mathfrak{Q}}\left(\alpha_{\imath \jmath} \mathrm{c}_{\jmath} \mathrm{c}_{\varkappa}^{\dagger}|\Omega\rangle-\beta_{\imath \jmath}^{*} \mathrm{c}_{\jmath}^{\dagger} \mathrm{c}_{\varkappa}^{\dagger}|\Omega\rangle\right) \\
& =\alpha_{\imath \varkappa}|\Omega\rangle-\sum_{\jmath \in \mathfrak{Q}} \beta_{\imath \jmath}^{*} \mathrm{c}_{\jmath}^{\dagger} \mathrm{c}_{\varkappa}^{\dagger}|\Omega\rangle
\end{aligned}
$$

and thereby

$$
\left\langle\Omega\left|\mathrm{a}_{\imath} \mathrm{c}_{\jmath}^{\dagger}\right| \Omega\right\rangle=\alpha_{\imath \jmath} \quad(\forall \imath, \jmath \in \mathfrak{Q})
$$

whence it follows that, as expected, the real and dimensionless Bogolyubov coefficients

$$
\alpha_{r A}(\hat{\mathrm{k}})=\frac{1}{2} e_{r}(\hat{\mathrm{k}}) \cdot \varepsilon_{A}(\hat{\mathrm{k}}) \frac{K_{A}+K}{\sqrt{ }\left(K_{A} K\right)}
$$

may be understood as the probability amplitudes that a Proca-Stückelberg pair of wave vector $\hat{k}$ is NOT CREATED out of the Fock vacuum $|0\rangle$ or, in other words, the RELAtive PERsistence PRoBABILITy amplitude for the ProcaStückelberg vacuum. In the very same manner the Bogolyubov coefficient $\alpha_{r, A}(\hat{\mathrm{k}})$ may be seen in turn as the relative persistence probability amplitude for the Chern-Simons vacuum $|\Omega\rangle$. In a similar way, by taking the in vacuum expectation value

$$
\left\langle 0\left|\Pi_{\imath} \mathrm{a}_{\varkappa}^{\dagger} \mathrm{c}_{\jmath}\right| 0\right\rangle=\sum_{\ell \in \mathfrak{Q}} \beta_{\jmath \ell}^{*}\left\langle 0\left|\frac{1}{2} \mathrm{a}_{\imath}^{2} \mathrm{a}_{\ell}^{\dagger} \mathrm{a}_{\varkappa}^{\dagger}\right| 0\right\rangle=\beta_{\jmath}^{*} \delta_{\varkappa \imath}(\forall \imath, \jmath, \varkappa \in \mathfrak{Q})
$$

it is also clear that we can understand the real Bogolyubov coefficient

$$
\beta_{r A}(\hat{\mathrm{k}})=\frac{1}{2} e_{r}(\hat{\mathrm{k}}) \cdot \varepsilon_{A}(\hat{\mathrm{k}}) \frac{K_{A}-K}{\sqrt{ }\left(K_{A} K\right)}
$$

as the relative probability amplitude that a squeezed pair of Proca-Stückelberg quanta is created out or absorbed into the Proca-Stückelberg vacuum. 
To proceed further on, consider e.g. the Chern-Simons quantum vector field and let us define

$$
\begin{array}{r}
\mathcal{S}(\theta, \widehat{\mathbf{n}}) \equiv \exp \{-i \theta \cdot \mathrm{T}(z, \bar{z}, \nu)\} \\
\theta \cdot \mathrm{T}(z, \bar{z}, \nu) \equiv \Pi^{\dagger}(\theta \bar{z})+\Pi(z \theta)+2 \mathrm{~N}(\theta \nu)
\end{array}
$$

where $\theta_{\ell}(\forall \ell \in \mathfrak{Q})$ is a real functional parameter, while the functional unit vector $\widehat{\mathbf{n}}_{\imath}$ is related to the functional parameters $z_{\imath}, \bar{z}_{\imath}, \nu_{\imath}$ through the relationship

$$
\widehat{\mathbf{n}}_{\imath}^{2}=\nu_{\imath}^{2}-z_{\imath} \bar{z}_{\imath}=1 \quad(\forall \imath \in \mathfrak{Q})
$$

For example, a suitable functional parametric form is provided by a set of hyperbolic and trigonometric variables $\left(v_{\imath}, \phi_{\imath}\right)$, in such a manner that

$$
\nu_{\imath}=\cosh v_{\imath} \quad z_{\imath}=\sinh v_{\imath} \exp \left\{-i \phi_{\imath}\right\} \quad\left(v_{\imath} \in \mathbb{R}, 0 \leq \phi_{\imath}<2 \pi, \forall \imath \in \mathfrak{Q}\right)
$$

From the basic commutation relation

$$
\left[\mathrm{T}(z, \bar{z}, \nu), \mathrm{c}_{\imath}\right]=-\bar{z}_{\imath} \mathrm{c}_{\imath}^{\dagger}-\nu_{\imath} \mathrm{c}_{\imath} \quad\left[\mathrm{T}(z, \bar{z}, \nu), \mathrm{c}_{\imath}^{\dagger}\right]=z_{\imath} \mathrm{c}_{\imath}+\nu_{\imath} \mathrm{c}_{\imath}^{\dagger}
$$

we readily find

$$
\left[\mathrm{T},\left[\mathrm{T}, \mathrm{c}_{\imath}\right]\right]=\mathrm{c}_{\imath} \quad\left[\mathrm{T},\left[\mathrm{T}, \mathrm{c}_{\imath}^{\dagger}\right]\right]=\mathrm{c}_{\imath}^{\dagger}
$$

As a consequence, we actually obtain the general Bogolyubov transformations in the form

$$
\begin{aligned}
& \mathrm{a}_{\imath}=\left(\cos \theta_{\imath}-i \nu_{\imath} \sin \theta_{\imath}\right) \mathrm{c}_{\imath}-i \mathrm{c}_{\imath}^{\dagger} \bar{z}_{\imath} \sin \theta_{\imath}=\alpha_{\imath} \mathrm{c}_{\imath}-\beta_{\imath}^{*} \mathrm{c}_{\imath}^{\dagger} \\
& \mathrm{a}_{\jmath}^{\dagger}=\left(\cos \theta_{\jmath}+i \nu_{\jmath} \sin \theta_{\jmath}\right) \mathrm{c}_{\jmath}^{\dagger}+i \mathrm{c}_{\jmath} z_{\jmath} \sin \theta_{\jmath}=\alpha_{\jmath}^{*} \mathrm{c}_{\jmath}^{\dagger}-\beta_{\jmath} \mathrm{c}_{\jmath}
\end{aligned}
$$

with

$$
\begin{array}{cc}
\alpha_{\jmath} \equiv \cos \theta_{\jmath}-i \nu_{\jmath} \sin \theta_{\jmath} & \beta_{\jmath}^{*} \equiv i \bar{z}_{\jmath} \sin \theta_{\jmath} \\
\widehat{\mathbf{n}}_{\jmath}^{2}=\nu_{\jmath}^{2}-\bar{z}_{\jmath} z_{\jmath}=1 & \left|\alpha_{\jmath}\right|^{2}+\left|\beta_{\jmath}\right|^{2}=1
\end{array}
$$

It follows thereby that the functional unitary operator

$$
\mathcal{S}(\theta, z, \nu)=\mathcal{S}(\theta, \widehat{\mathbf{n}})=\mathcal{S}(\alpha, \beta) \quad \mathcal{S}^{-1}=\mathcal{S}^{\dagger}
$$

does generate the Bogolyubov similarity transformations which connect the Proca-Stückelberg and Chern-Simons vector fields on the boundary hyper-plane $\zeta \cdot x=0$ according to the suitable definitions

$$
A_{\mathrm{PS}}^{\nu}(\hat{\mathrm{x}})=\mathcal{S}^{-1} A_{\mathrm{CS}}^{\nu}(\hat{\mathrm{x}}) \mathcal{S} \quad|\mathrm{CS}\rangle=\mathcal{S}|\mathrm{PS}\rangle
$$

Moreover we obtain

$$
\begin{aligned}
A_{\mathrm{PS}}^{\nu}(\hat{\mathrm{x}}) & =\sum_{\imath \in \mathfrak{Q}} \mathcal{S}^{-1}\left[\mathrm{c}_{\imath} v_{\imath}(\hat{\mathrm{x}})+\mathrm{c}_{\imath}^{\dagger} v_{\imath}^{*}(\hat{\mathrm{x}})\right] \mathcal{S} \\
& =\sum_{\jmath \in \mathfrak{Q}}\left[\mathrm{a}_{\jmath} u_{\jmath}(\hat{\mathrm{x}})+\mathrm{a}_{\jmath}^{\dagger} u_{\jmath}^{*}(\hat{\mathrm{x}})\right] \\
A_{\mathrm{CS}}^{\nu}(\hat{\mathrm{x}}) & =\sum_{\imath \in \mathfrak{Q}} \mathcal{S}\left[\mathrm{a}_{\imath} u_{\imath}(\hat{\mathrm{x}})+\mathrm{a}_{\imath}^{\dagger} u_{\imath}^{*}(\hat{\mathrm{x}})\right] \mathcal{S}^{-1} \\
& =\sum_{\jmath \in \mathfrak{Q}}\left[\mathrm{c}_{\jmath} v_{\jmath}(\hat{\mathrm{x}})+\mathrm{c}_{\jmath}^{\dagger} v_{\jmath}^{*}(\hat{\mathrm{x}})\right]
\end{aligned}
$$

where, in the case of the parity breaking medium and Minkowski space in four dimensions, we have e.g.

$$
u_{\imath}(\hat{\mathrm{x}}) \equiv u_{\hat{\mathbf{k}}, r}^{\nu}(x=0, \hat{\mathrm{x}}) \quad v_{\jmath}(\hat{\mathrm{x}}) \equiv v_{\hat{\mathbf{p}}, A}^{\nu}(x=0, \hat{\mathrm{x}})
$$

Hence, the general Bogolyubov transformation is nothing but a functional rotation in the Fock space with parameter functions $(\theta, z, \nu)=(\theta, \widehat{\mathbf{n}})=(\alpha, \beta)$, the generators of which are the squeezed pairs emission $\Pi^{\dagger}(\theta \bar{z})$, the squeezed pairs absorption $\Pi(\theta z)$ and the squeezed pairs number $\mathrm{N}(\theta \nu)$ operators, which actually fulfill the functional commutation relations arising from the $\mathrm{SU}(2)$ Lie algebra. 


\section{THE EXTENDED SAUTER-SCHWINGER-NIKISHOV FORMULA}

Suppose that at some initial time $t_{\imath}$ our system is in a definite state, e.g. the vacuum $\mid 0$ in $\rangle$ as defined in equation (85), so that it does not contain any Proca-Stückelberg vector particles, and that particle detectors are homogeneously distributed and placed within a small but finite spatial volume $\Delta v$. Then the final state at some later although close time $\mathrm{t}_{f}$, with $t_{f}-t_{\imath}=\Delta t$, has some calculable probability to contain zero, one, two, etc. emitted squeezed pairs of vector particles of the Proca-Stückelberg or Chern-Simons particles. For example the LIV vacuum persistence probability amplitude, i.e., the probability amplitude of no emission of squeezed Chern-Simons pairs at the time $\mathrm{t}_{f}$, will be given by

$$
\langle\Omega \text { out }| 0 \text { in }\rangle=\left\langle 0 \text { out }\left|\mathcal{S}^{\dagger}\right| 0 \text { in }\right\rangle
$$

For this interpretation to make sense, one has to actually verify that the vacuum to vacuum transition probability

$$
\left.W_{0, f \leftarrow \imath} \equiv \mid\langle\Omega \text { out }| 0 \text { in }\right\rangle\left.\right|^{2}
$$

is not greater than one. To this concern consider the Hermitean operators which correspond to the number of CS and PS quanta with quantum numbers $\imath \in \mathfrak{Q}$ : namely,

$$
\mathrm{N}_{\imath}^{\mathrm{CS}} \equiv \frac{1}{2}\left(\mathrm{c}_{\imath}^{\dagger} \mathrm{c}_{\imath}+\mathrm{c}_{\imath} \mathrm{c}_{\imath}^{\dagger}\right)=\mathcal{S} \mathrm{N}_{\imath}^{\mathrm{PS}} \mathcal{S}^{-1}=\mathrm{N}_{\imath}^{\mathrm{PS}}+\alpha_{\imath} \beta_{\imath}^{*} \Pi_{\imath}^{\dagger}+\alpha_{\imath}^{*} \beta_{\imath} \Pi_{\imath}
$$

where

$$
\Pi_{\imath} \equiv \mathrm{a}_{\imath}^{2} \quad 4\left|\alpha_{\jmath} \beta_{\jmath}^{*}\right|^{2}+\left(\left|\alpha_{\jmath}\right|^{2}-\left|\beta_{\jmath}\right|^{2}\right)^{2}=1
$$

It follows that if we set

$$
\xi_{\jmath} \equiv 2 \alpha_{\jmath} \beta_{\jmath}^{*}, \quad w_{\jmath} \equiv\left|\alpha_{\jmath}\right|^{2}-\left|\beta_{\jmath}\right|^{2}
$$

we can write the following squeeze pair operator Golden Rule [39] that actually occurs $\forall \imath, \jmath, \ell, \ldots \in \mathfrak{Q}$ :

$$
2 \mathrm{~N}_{\imath}^{\mathrm{CS}}=\xi_{\imath} \Pi_{\imath}+\bar{\xi}_{\imath} \Pi_{\imath}^{\dagger}+2 w_{\imath} \mathrm{N}_{\imath}^{\mathrm{PS}} \quad \bar{\xi}_{\imath} \xi_{\imath}+w_{\imath}^{2}=1
$$

It is important to remark that the above equality (124) holds true thanks to the similarity property satisfied by the Bogolyubov coefficients $\alpha_{\imath}, \beta_{\jmath}$. Then, for any complex distribution function $\varphi(\hat{k} ; A, r)=\varphi_{\imath}$, we can write

$$
\begin{aligned}
2 \mathrm{~N}^{\mathrm{CS}}(\varphi) & \equiv \sum_{\imath \in \mathfrak{Q}} 2 \mathrm{~N}_{\imath}^{\mathrm{CS}} \varphi_{\imath}=\sum_{\imath \in \mathfrak{Q}}\left\{\mathrm{c}_{\imath}^{\dagger}, \mathrm{c}_{\imath}\right\} \varphi_{\imath} \\
& =\Pi^{\dagger}(\bar{\xi} \varphi)+\Pi(\xi \varphi)+2 \mathrm{~N}^{\mathrm{PS}}(w \varphi)=\varphi \cdot \mathrm{T}(\xi, \bar{\xi}, w)
\end{aligned}
$$

In the application of the above general setting to the present circumstance, it should be kept in mind that, owing to the presence of the boundary $\zeta \cdot \mathrm{x}=0$, then translation invariance holds true only in the time evolution and in the transverse foliation $\zeta \cdot \mathrm{x}=$ constant. It follows thereby that we can write

$$
\begin{aligned}
\left.2 \mathrm{~N}_{\imath}^{\mathrm{CS}} \mid 0 \text { in }\right\rangle & \left.\left.=\left[\mathrm{a}_{\imath}, \mathrm{a}_{\imath}^{\dagger}\right] \mid 0 \text { in }\right\rangle=\delta_{\imath \imath} \mid 0 \text { in }\right\rangle \\
& \left.\left.=\delta^{(3)}(0) \mid 0 \text { in }\right\rangle \equiv \Delta \sigma \Delta t(2 \pi)^{-3} \mid 0 \text { in }\right\rangle
\end{aligned}
$$

where $\Delta \sigma$ denotes the small unit area in the $O y z$-plane - e.g. a section of a region in the 3 -dimensional space where the particle detectors are placed. Thus we eventually obtain

$$
\begin{aligned}
\langle\Omega \text { out }| 0 \text { in }\rangle & =\left\langle 0 \text { out }\left|\mathcal{S}_{\varphi}^{-1}\right| 0 \text { in }\right\rangle \\
& =\left\langle 0 \text { out }\left|\exp \left\{2 i \mathrm{~N}^{\mathrm{CS}}(\varphi)\right\}\right| 0 \text { in }\right\rangle \\
& =\prod_{\imath \in \mathfrak{Q}} \exp \left\{i \varphi_{\imath} \Delta \sigma \Delta t(2 \pi)^{-3}\right\} \\
& =\exp \left\{i \Delta \sigma \Delta t(2 \pi)^{-3} \sum_{\imath \in \mathfrak{Q}} \varphi_{\imath}\right\}
\end{aligned}
$$

However, according to the natural interpretation which arises from eq. (107), it is mandatory for consistency to identify

$$
\varphi_{\jmath} \equiv i \ln \alpha_{\jmath}^{*}=\operatorname{Arg} \alpha_{\jmath}+\frac{1}{2} i \ln \left|\alpha_{\jmath}\right|^{2} \quad(\forall \jmath \in \mathfrak{Q})
$$


As a matter of fact, according to Nikishov [40], the logarithm of vacuum to vacuum transition amplitude is provided by

$$
\ln \langle\Omega \text { out }| 0 \text { in }\rangle=-\Delta \sigma \Delta t(2 \pi)^{-3} \sum_{\imath \in \mathfrak{Q}} \ln \alpha_{\imath}^{*}
$$

As a consequence it is possible to express the out vacuum in terms of the in operators in the explicit form

$$
\begin{aligned}
\langle\Omega \text { out }| & =\langle 0 \text { out }| \mathcal{S}_{\varphi}^{-1} \\
& =\langle 0 \text { out }| \exp \left\{2 i \mathrm{~N}^{\mathrm{CS}}(\varphi)\right\} \\
& =\langle 0 \text { out }| \exp \left\{-2 \mathrm{~N}^{\mathrm{CS}}\left(\ln \alpha^{*}\right)\right\} \\
& =\langle 0 \text { out }| \exp \left\{-\sum_{\imath \in \mathfrak{Q}} \mathrm{c}_{\imath}^{\dagger} \mathrm{c}_{\imath} \ln \alpha_{\imath}^{*}\right\}
\end{aligned}
$$

whence it immediately follows that, by the very construction,

$$
\left.\mathrm{c}_{\varkappa} \mid 0 \text { out }\right\rangle=0 \quad(\forall \varkappa \in \mathfrak{Q})
$$

Notice that the non-singular operator $\mathcal{S}_{\varphi}$ is not unitary, owing to the presence of an imaginary part in the distribution function $\varphi=i \ln \alpha^{*}$. Furthermore, from the golden operator identity (124) it follows that we can write

$$
2 \mathcal{N}(\varphi)=\varphi \cdot \mathrm{T}(\xi, \bar{\xi}, w) \equiv \Pi^{\dagger}(\varphi \bar{\xi})+\Pi(\xi \varphi)+2 \mathrm{~N}(\varphi w)
$$

in such a manner that the out vacuum state can be expressed $\grave{a}$ la Dirac as an infinite sea of squeezed pairs, i.e., a coherent like state involving any number of squeezed pairs of any quantum numbers: namely,

$$
\begin{aligned}
& \langle\Omega \text { out }|=\langle 0 \text { out }| \mathcal{S}_{\varphi}^{-1}=\langle 0 \text { out }| \exp \left\{-2 \mathcal{N}\left(\ln \alpha^{*}\right)\right\} \\
& =\langle 0 \text { out }| \exp \left\{-\Pi^{\dagger}\left(\bar{\xi} \ln \alpha^{*}\right)-\Pi\left(\xi \ln \alpha^{*}\right)-2 \mathrm{~N}\left(w \ln \alpha^{*}\right)\right\}
\end{aligned}
$$

in which

$$
\xi_{\jmath} \equiv 2 \alpha_{\jmath} \beta_{\jmath}^{*} \quad w_{\jmath} \equiv\left|\beta_{\jmath}\right|^{2}-\left|\alpha_{\jmath}\right|^{2} \quad(\forall \jmath \in \mathfrak{Q})
$$

Finally, one can readily generalize to the present circumstance the case of spinor QED in the presence of a uniform electric field on the four dimensional Minkowski space: actually we have

$$
\langle\Omega \text { out }| 0 \text { in }\rangle=\exp \left\{-i \Delta \sigma \Delta t(2 \pi)^{-3} \sum_{A} \int \mathrm{d} \hat{k} \varphi(\alpha)\right\}
$$

with

$$
\hat{k} \equiv\left(\omega, k_{y}, k_{z}\right) \quad \alpha(\hat{k}, A) \equiv \frac{1}{2} \cdot \frac{K_{A}+K}{\sqrt{ }\left(K K_{A}\right)} \quad(A= \pm, L)
$$

where $K$ and $K_{A}$ are provided by equation (61), in such a manner that we can eventually write

$$
\begin{aligned}
\mid\langle\Omega \text { in }| 0 \text { out }\rangle\left.\right|^{2} & =\exp \left\{-\Delta \sigma \Delta t(2 \pi)^{-3} \sum_{A} \int \mathrm{d} \hat{\mathrm{k}} \ln |\alpha(\hat{\mathrm{k}}, A)|^{2}\right\} \\
& =\exp \left\{-2 \Delta \sigma \Delta t(2 \pi)^{-3} \sum_{A= \pm} \int \mathrm{d} \hat{\mathrm{k}} \ln \alpha(\hat{\mathrm{k}}, A)\right\}
\end{aligned}
$$

which is nothing but the suitable generalization of celebrated Sauter-Schwinger formula [41] to the present context of a transition across a parity breaking medium from the Minkowski space and viceversa. For example, to the leading order in the LIV relevant scales $\zeta \ll m \ll M$, which properly characterize the present framework, explicit evaluation shows - see Appendix - that the logarithm of the vacuum persistence probability trough the boundary per unit time and unit transverse area is actually approximately given by

$$
\left.\frac{-1}{\Delta t \Delta \sigma} \ln \mid\langle\Omega \text { in }| 0 \text { out }\right\rangle \mid \approx \zeta \frac{m M c^{3}}{16 \pi^{2} \hbar^{2}}\left\{1+\frac{\hbar \zeta}{2 m c}\left(2 \ln \frac{M}{m}-\ln \frac{\hbar \zeta}{2 m c}\right)+O\left(\frac{\hbar \zeta}{m c}\right)\right\}
$$

Notice that, as expected, we recover the null result in the Lorentz invariant limit $\zeta \rightarrow 0$ when a unique cyclic vacuum state is left in the Fock space, while the vacuum persistence probability becomes not completely negligible for $\zeta \Delta t \Delta \sigma m M c^{3} \simeq 8 \pi^{2} \hbar^{2}$. 


\section{CONCLUSION}

The problem of propagation of vector particles through a boundary between a parity breaking medium and an empty vacuum is quite interesting as it may arise in heavy ion and astro- physics. In this paper we restricted ourselves with the Chern-Simons dynamics generated by a CS vector orthogonal to the flat boundary which perfectly guaranties the gauge invariance while provides the Lorentz symmetry violation in the Maxwell-Chern-Simons part coherent with boundary implementation. On the other half-space we have a massive quantum vector field in vacuum. We investigated the different quantum field aspects of the model described before at quasi-classical level. In particular, the transmission/reflection coefficients for different cases were found in [48, 49] and the possible influence on the cooling rate of neutron star was shown in [27].

However, the results of the present work allow us to describe the particle transition through the boundary between areas with different field equations at quantum level in a more general form. The fact that Bogolyubov transformations may be rewritten as a functional rotation in the Fock space is useful in problems not only connected with MCS electrodynamics in a finite volume, but in other investigations concerned with different Fock spaces for geometrically and physically different space-time regions and propagation between them.

We draw attention of a reader also to the probability of inducing finite volume bubbles of distinct matter/radiation background undertaken in the Section 5 and in the Appendix. It looks useful for description of phase transition to and from the parity breaking media in heavy ion collisions. However we stress that for infinite volume subspaces the total transition probability vanishes and the two media coexist. This is justified by calculation of the probability to find any number of squeezed pairs of vector particles of the Proca-Stuckelberg or Chern-Simons kind in crossing the boundary between the empty space-time and the parity breaking medium.

\section{ACKNOWLEDGEMENTS}

A.A. and S.K. were supported by Grant RFBR projects 16-02-00348 and also got a financial support of SPbSU. A.A. was also supported through grants FPA2013-46570, 2014-SGR-104 and Consolider CPAN. As well funding was partially provided by the Spanish MINECO under project MDM-2014-0369 of ICCUB (Unidad de Excelencia 'Maria de Maeztu'). This collaboration has been partially supported by grants from I.N.F.N. Sezione di Bologna Inviti 2015 and RFO 2014 DIFA Universitá di Bologna.

\section{APPENDIX A: PROBABILITY FLUX}

In this Appendix we will calculate, to the leading approximation in the relevant physical parameters of the present model, the probability flux from the Proca-Stückelberg vacuum state $|0\rangle$ to the Chern-Simons vacuum state $|\Omega\rangle$ and viceversa, due to transmission and reflection of squeezed pairs quanta through the boundary that separates the parity breaking medium from the Minkowski empty space. To start with, we have to invert the Chern-Simons frequency formula for $\hat{\mathrm{k}}^{2}>m^{2}$ and $\zeta^{\lambda}=(0, \zeta, 0,0)(\zeta>0)$ : we find

$$
\begin{aligned}
& \omega_{\mathbf{k}, \pm}=\sqrt{\mathbf{k}^{2}+m^{2}+\frac{1}{2} \zeta^{2} \pm \zeta \sqrt{k_{x}^{2}+m^{2}+\frac{1}{4} \zeta^{2}}} \\
& \hat{\mathrm{k}}^{2}-K^{2}-m^{2}-\frac{1}{2} \zeta^{2}=\zeta \sqrt{K^{2}+m^{2}+\frac{1}{4} \zeta^{2}} \Longleftrightarrow\left\{K^{2}-\left(\hat{\mathrm{k}}^{2}-m^{2}\right)\right\}^{2}-\hat{\mathrm{k}}^{2} \zeta^{2}=0 \\
& K^{2}-\left(\hat{\mathrm{k}}^{2}-m^{2}\right)= \pm \sqrt{\hat{\mathrm{k}}^{2} \zeta^{2}} \Longleftrightarrow K_{ \pm}(\hat{\mathrm{k}})=\sqrt{\hat{\mathrm{k}}^{2}-m^{2} \pm \zeta \sqrt{\hat{\mathrm{k}}^{2}}}
\end{aligned}
$$

As we have already emphasized the general requirements $k_{-}^{2}>0 \vee K_{-}(\hat{\mathrm{k}})>0$ entail the conservative lower and upper approximate bounds for angular frequency

$$
m\left(1+\frac{\epsilon}{2}\right) \lesssim \omega \lesssim M \quad \epsilon=\frac{\hbar \zeta}{m c}
$$

where, as already mentioned, the UV cut-off $M$ is the natural albeit model-dependent axion-like decay constant size, typically ranging within $10^{9} \div 10^{17} \mathrm{GeV}$, according to the recent estimates - see e.g. the review paper [44]. If we suppose here that e.g. $\zeta \ll m$, then we have for $\epsilon=(\zeta / m) \ll 1$

$$
K(\hat{\mathrm{k}})=\sqrt{\hat{\mathrm{k}}^{2}-m^{2}}>0 \quad \text { for } \quad \hat{\mathrm{k}}^{2}>m^{2}
$$




$$
\begin{gathered}
K_{ \pm}(\hat{\mathrm{k}})=K(\hat{\mathrm{k}}) \sqrt{1 \pm \chi(\hat{\mathrm{k}})}>0 \quad \text { for } \quad \hat{\mathrm{k}}^{2}>m^{2}\left[1+\epsilon^{2}\left(1 \pm \sqrt{\frac{1}{\epsilon^{2}}+\frac{1}{4}}\right)\right] \\
\chi(\hat{\mathrm{k}})=\frac{\zeta \sqrt{\hat{\mathrm{k}}^{2}}}{\hat{\mathrm{k}}^{2}-m^{2}} \lesssim 1 \quad \text { for } \quad \hat{\mathrm{k}}^{2} \gtrsim m^{2}(1+\epsilon)
\end{gathered}
$$

Notice that this particular function $\chi(\hat{\mathrm{k}})$ is a monotonically decreasing function for $\hat{\mathrm{k}}^{2} \gtrsim m^{2}(1+\epsilon)$ which is bounded by $O(1)$ because

$$
\frac{\hat{\mathrm{k}}}{\hat{\mathrm{k}}^{2}} \cdot \nabla_{\hat{\mathrm{k}}} \chi(\hat{\mathrm{k}})=-\frac{\zeta}{\sqrt{\hat{\mathrm{k}}^{2}}} \cdot \frac{\hat{\mathrm{k}}^{2}+m^{2}}{\left(\hat{\mathrm{k}}^{2}-m^{2}\right)^{2}}<0 ; \quad \chi(\hat{\mathrm{k}}=m \sqrt{1+\epsilon})=\frac{\zeta \sqrt{1+\epsilon}}{m \epsilon}=1+\frac{\epsilon}{2}+\ldots
$$

Then the Bogolyubov transmission coefficient can be recast under the suitable form

$$
\alpha(\hat{\mathrm{k}})_{ \pm} \equiv \frac{1}{2} \cdot \frac{K_{ \pm}+K}{\sqrt{ }\left(K K_{ \pm}\right)}=\frac{1+\sqrt{1 \pm \chi(\hat{\mathrm{k}})}}{2 \sqrt[4]{1 \pm \chi(\hat{\mathrm{k}})}}=\frac{1}{2 \sqrt[4]{1 \pm \chi(\hat{\mathrm{k}})}}+\frac{1}{2} \sqrt[4]{1 \pm \chi(\hat{\mathrm{k}})}
$$

while the integration volume in Fourier space is correspondingly bounded by

$$
\int \mathrm{d} \hat{\mathrm{k}} \equiv \int_{-M}^{M} \mathrm{~d} \omega \theta\left(\omega^{2}-m^{2}(1+\epsilon)\right) \iint_{-\infty}^{\infty} \mathrm{d} k_{y} \mathrm{~d} k_{z} \theta\left(\omega^{2}-m^{2}(1+\epsilon)-k_{x}^{2}-k_{y}^{2}\right)
$$

Then we can approximate by keeping the leading term

$$
\begin{aligned}
\ln \alpha(\hat{\mathrm{k}}) & =\ln [1+\sqrt{1 \pm \chi(\hat{\mathrm{k}})}]-\ln 2-\frac{1}{4} \ln [1 \pm \chi(\hat{\mathrm{k}})] \\
& = \pm \frac{1}{4} \chi(\hat{\mathrm{k}})-\frac{1}{2} \cdot \frac{1}{8} \chi^{2}(\hat{\mathrm{k}})-\frac{1}{32} \chi^{2}(\hat{\mathrm{k}})+\cdots \mp \frac{1}{4} \chi(\hat{\mathrm{k}})+\frac{1}{8} \chi^{2}(\hat{\mathrm{k}})+\cdots \\
& \approx \frac{1}{32} \chi^{2}(\hat{\mathrm{k}}) \quad \text { for } \quad \chi(\hat{\mathrm{k}})<1
\end{aligned}
$$

within the phase space volume. Thus we approximately obtain

$$
\int \mathrm{d} \hat{\mathrm{k}} \ln \alpha(\hat{\mathrm{k}}) \approx \frac{\zeta^{2}}{16} \int_{0}^{M} \mathrm{~d} \omega \theta\left(\omega^{2}-m^{2}(1+\epsilon)\right) \iint_{-\infty}^{\infty} \mathrm{d} k_{y} \mathrm{~d} k_{z} \theta\left(\hat{\mathrm{k}}^{2}-m^{2}(1+\epsilon)\right) \frac{\hat{\mathrm{k}}^{2}}{\left(\hat{\mathrm{k}}^{2}-m^{2}\right)^{2}}
$$

and if we set $q=k_{y}^{2}+k_{z}^{2}$ after turning to planar polar coordinates we obtain

$$
\int \mathrm{d} \hat{\mathrm{k}} \ln \alpha(\hat{\mathrm{k}}) \approx \frac{\pi \zeta^{2}}{16} \int_{0}^{M} \mathrm{~d} \omega \theta\left(\omega^{2}-m^{2}(1+\epsilon)\right) \int_{0}^{\infty} \mathrm{d} q \theta\left(\omega^{2}-m^{2}(1+\epsilon)-q\right) \frac{\omega^{2}-q}{\left(\omega^{2}-m^{2}-q\right)^{2}}
$$

To evaluate the above integral it is convenient to set

$$
v=\omega^{2}-m^{2}(1+\epsilon) \quad a=\sqrt{1+\epsilon} \quad b=\frac{M}{m}
$$

in such a manner that we can write

$$
\begin{aligned}
\int \mathrm{d} \hat{\mathrm{k}} \ln \alpha(\hat{\mathrm{k}}) & \left.\approx \frac{\pi \zeta^{2}}{16} \int_{0}^{M} \mathrm{~d} \omega \theta(v) \frac{\partial}{\partial \xi} \int_{0}^{v} \mathrm{~d} q \frac{\omega^{2}-q}{v-q+\epsilon m^{2}-\xi}\right|_{\xi=0} \\
& =\frac{\pi \zeta^{2}}{16} \int_{0}^{M} \mathrm{~d} \omega \theta(v) \frac{\partial}{\partial \xi}\left\{\left(m^{2}+\xi\right)\left[\ln \left(v+\epsilon m^{2}-\xi\right)-\ln \left(\epsilon m^{2}-\xi\right)\right]\right\}_{\xi=0} \\
& =\frac{\pi}{16} m^{3} \epsilon^{2} \int_{a}^{b} \mathrm{~d} x\left[\ln (1+\varkappa)+\frac{1}{\epsilon} \cdot \frac{\varkappa}{1+\varkappa}\right]
\end{aligned}
$$

where we have set

$$
\varkappa=\frac{v}{\epsilon m^{2}}=\frac{\omega^{2}-m^{2}(1+\epsilon)}{\epsilon m^{2}}=\frac{1}{\epsilon}\left(x^{2}-1\right)-1
$$


Then we find

$$
\int \mathrm{d} \hat{\mathrm{k}} \ln \alpha(\hat{\mathrm{k}}) \approx \frac{\pi}{16} m^{3} \epsilon^{2} \int_{a}^{b} \mathrm{~d} x\left[\ln (x+1)+\ln (x-1)-\ln \epsilon+\frac{1}{\epsilon}+\frac{1}{2}\left(\frac{1}{x+1}-\frac{1}{x-1}\right)\right]
$$

so that, taking the assumption $0 \leq \epsilon \ll 1 \ll b$ properly into account,

$$
\begin{aligned}
\int \mathrm{d} \hat{\mathrm{k}} \ln \alpha(\hat{\mathrm{k}}) & \approx \frac{\pi b}{16} m^{3} \epsilon^{2}\left\{\frac{1}{\epsilon}+2 \ln b-\ln \epsilon+O(1)\right\} \\
& \approx \frac{\pi b}{16} m^{3} \epsilon\left\{1+\epsilon \ln \frac{b^{2}}{\epsilon}+O(\epsilon)\right\}
\end{aligned}
$$

where we have neglected also by terms of order $\ln (\epsilon) / b<O(1)$ Thereby, under the assumption $M \gg m \gg \zeta$, we eventually find that, to the leading order in the relevant LIV numbers $\epsilon \ll 1$ and $b \gg 1$, the dominant contribution to the vacuum to vacuum persistence probability is provided by the flux density

$$
\left.\frac{1}{\Delta t \Delta \sigma} \ln \mid\langle\Omega \text { in }| 0 \text { out }\right\rangle \mid \approx-\zeta \frac{m M c^{3}}{16 \pi^{2} \hbar^{2}}\left\{1+\frac{\hbar \zeta}{2 m c}\left(2 \ln \frac{M}{m}-\ln \frac{\hbar \zeta}{2 m c}\right)+O\left(\frac{\hbar \zeta}{m c}\right)\right\}
$$

where we have taken into account a factor two, because both LIV polarization contribute the same amount to the leading order in this approximation.

[1] S.M. Carroll, G.B. Field and R. Jackiw, Phys. Rev. D 41, 1231 (1990).

[2] G. M. Shore, Nucl. Phys. B 717, 86 (2005).

[3] T. Jacobson, S. Liberati and D. Mattingly, Ann. Phys. (NY) 321 (2006) 150.

[4] V. A. Kostelecký and N. Russell, Rev. Mod. Phys. 83, 11 (2011).

[5] W. Bietenholz, arXiv:0806.3713 [hep-ph].

[6] F. W. Stecker and S. T. Scully, New J. Phys. 11, 085003 (2009).

[7] A. A. Andrianov and R. Soldati, Phys. Rev. D 51, 5961 (1995).

[8] D. Colladay and V. A. Kostelecký, Phys. Rev. D 55, 6760 (1997).

[9] D. Colladay and V. A. Kostelecký, Phys. Rev. D 58, 116002 (1998).

[10] A. A. Andrianov and R. Soldati, Phys. Lett. B 435, 449 (1998).

[11] S. R. Coleman and S. L. Glashow, Phys. Lett. B 405, 249 (1997).

[12] S. R. Coleman and S. L. Glashow, arXiv:9808446 [hep-ph].

[13] S. R. Coleman and S. L. Glashow, Phys. Rev. D 59 (1999) 116008; arXiv:9812418 [hep-ph].

[14] R. C. Myers and M. Pospelov, Phys. Rev. Lett. 90, 211601 (2003).

[15] R. Montemayor and L. F. Urrutia, Phys. Rev. D 72, 045018 (2005).

[16] J. Alfaro, A.A. Andrianov, M. Cambiaso, P. Giacconi and R. Soldati, Int. J. Mod. Phys. A 25, 3271 (2010); Phys. Lett. B 639, 586 (2006).

[17] M. Cambiaso, R. Lehnert and R. Potting, Phys. Rev. D 85, 085023 (2012).

[18] V. A. Kostelecký and M. Mewes, Phys. Rev. D 66, 056005 (2002).

[19] G. M. Shore, Contemp. Phys. 44, 503 (2003).

[20] H. C. Cheng, M. A. Luty, S. Mukohyama and J. Thaler, JHEP 0605, 076 (2006).

[21] A. A. Andrianov, R. Soldati and L. Sorbo, Phys. Rev. D 59, 025002 (1998).

[22] N. Arkani-Hamed, H. C. Cheng, M. A. Luty and S. Mukohyama, JHEP 0405, 074 (2004).

[23] N. Arkani-Hamed, H. C. Cheng, M. Luty and J. Thaler, JHEP 0507, 029 (2005).

[24] L. Abbott and P. Sikivie, Phys. Lett. B 120, 133 (1983).

[25] M. Kuster, G. Raffelt and B. Beltran (eds), Axions: Theory, Cosmology and Experimental Searches, Lecture Notes in Physics 741 (Springer, 2008).

[26] E. W. Kolb and M. S. Turner, The Early Universe (Westview Press, 1990); Y. Sofue and V. Rubin, Ann. Rev. Astron. Astrophys.39 (2001) 137-174; S. J. Asztalos et al., Ap. Jour. 571, L27 (2002) .

[27] A.A. Andrianov, V.A. Andrianov, Domenec Espriu and S.S. Kolevatov, Eur. Phys. J. C 76, 169 (2016).

[28] Peter Wurm for the CERES Collaboration, Nucl. Phys. A 590, 103c (1995); G. Agakichiev et al. (CERES Collaboration), Eur. Phys. J. C 4, 231 (1998); R. Arnaldi R. et al. (NA60 Collaboration), Phys. Rev. Lett. 96 (2006) 162302.

[29] A. Adare et al. (PHENIX Collaboration), Phys. Rev. C 81, 034911 (2010).

[30] G. Agakichiev et al. (HADES Collaboration), Phys. Rev. Lett. 98, 052302 (2007); Phys. Lett. B 663, 43 (2008).

[31] I.Tserruya, Electromagnetic Probes, arXiv:0903.0415 [nucl-ex].

[32] A. A. Andrianov, V. A. Andrianov, D. Espriu and X. Plannels, Phys. Lett. B 710, 230 (2012).

[33] A. A. Andrianov and D. Espriu, Phys. Lett. B 663, 450 (2008); A. A. Andrianov, D. Espriu and V. A. Andrianov, Phys. Lett. B 678, 416 (2009). 
[34] D. Kharzeev, R. D. Pisarski and M. H. G. Tytgat, Phys. Rev. Lett. 81, 512 (1998); K. Buckley, T. Fugleberg, and A. Zhitnitsky, Phys. Rev. Lett. 84, 4814 (2000); D. Kharzeev, Phys. Lett. B633, 260 (2006); D. E. Kharzeev, L. D. McLerran and H. J. Warringa, Nucl. Phys. A 803, 227 (2008).

[35] P. V. Buividovich, M. N. Chernodub, E. V. Luschevskaya, M. I. Polikarpov, Phys. Rev. D 80, 054503 (2009).

[36] B. I. Abelev et al. (STAR Collaboration), Phys. Rev. Lett. 103, 251601 (2009); S. A. Voloshin, J. Phys. Conf. Ser. 230, 012021 (2010).

[37] N. D. Birrell and P. C. W. Davies, Quantum Fields in Curved Space (Cambridge University Press, Cambridge (UK), 1982).

[38] A. A. Andrianov, S. S. Kolevatov and R. Soldati, Theor. Math. Phys., 184, 1213 (2015).

[39] Roberto Soldati, J. Phys. A 44, 305401 (2011).

[40] A. I. Nikishov, Sov. Phys. - JETP 30, 660 (1970); English translation from Zh. Eksp. Teor. Fiz. 57, 1210 (1969); N.B. Narozhnyi and A.I. Nikishov, Sov. J. Nucl. Phys. 11, 596 (1970), English translation from Yad. Fiz. 11, 1072 (1970).

[41] F. Sauter, Z. Phys. 69, 742 (1931); W. Heisenberg and H. Euler, Z. Phys. 98, 714 (1936); English translation at e-Print arXiv:0605038 [physics]; J. Schwinger, Phys. Rev. 82, 664 (1951).

[42] E. C. G. Stueckelberg, Helv. Phys. Acta 30, 209 (1957).

[43] I. A. Batalin and E. S. Fradkin, Nucl. Phys. B 279, 514 (1987).

[44] Rouven Essig et al., Report of the Community Summers Study 2013 (Snowmass), arXiv:1311.0029 [hep-ph].

[45] A. A. Andrianov, D. Espriu, P. Giacconi and R. Soldati, JHEP 09, 057 (2009).

[46] A. A. Andrianov, D. Espriu, F. Mescia and A. Renau, Phys. Lett. B 684, 101 (2010).

[47] A.A. Andrianov, P. Giacconi and R. Soldati, JHEP 02, 030 (2002).

[48] A. A. Andrianov, S. S. Kolevatov and R. Soldati, JHEP 11, 007 (2011).

[49] A. A. Andrianov and S. S. Kolevatov, Theor. Math. Phys. 175, 744 (2013); English translation from Teor. Mat. Fiz. 175, $357(2013)$ 